\title{
CONTINUOUS LIMITS OF DISCRETE PERIMETERS
}

\author{
Antonin Chambolle ${ }^{1}$, Alessandro Giacomini $^{2}$ And Luca Lussardi ${ }^{3}$
}

\begin{abstract}
We consider a class of discrete convex functionals which satisfy a (generalized) coarea formula. These functionals, based on submodular interactions, arise in discrete optimization and are known as a large class of problems which can be solved in polynomial time. In particular, some of them can be solved very efficiently by maximal flow algorithms and are quite popular in the image processing community. We study the limit in the continuum of these functionals, show that they always converge to some "crystalline" perimeter/total variation, and provide an almost explicit formula for the limiting functional.
\end{abstract}

Mathematics Subject Classification. 49Q20, 65K10.

Received February 27, 2009.

Published online December 16, 2009.

\section{INTRODUCTION}

In the past ten years, optimization methods for image processing task have made a lot of progress, thanks to the development of combinatorial methods (maximal flow/minimal cut, and other graph-based optimization methods - see for instance [11], and [1,4] and the references therein). These methods are not new, the idea of representing Ising energies (i.e., discrete approximations of perimeters) on graphs and computing minimum points using maximal flows algorithms dates back at least to the 70s [14]. However, the evolution of computers and the development of new algorithms [4], oriented towards specific applications, have contributed a lot towards the recent increase of activity in this field. In image processing, the idea is to regularize ill-posed inverse problems for finding sets (shapes) or partitions into labels of an image, by penalizing a discrete variant of their perimeter. We try to consider, in this paper, the most general energies which can be tackled by these methods, and even a little bit more: we consider discrete submodular energies (see Eq. (1.1) below), defined on discrete subsets of a finite lattice $\mathcal{V} \subset h \mathbb{Z}^{N}, h>0$, for which it is known that polynomial algorithms do exist (see for instance $[7,12,15]$ ). We will show that, appropriately extended into functions of general vectors in $\mathbb{R}^{\mathcal{V}}$ by means of a generalized coarea formula, these energies are, in fact, convex. This is already known (although our setting is a bit different, as well as our proofs which apply to other situations, including functionals defined in the continuous setting) in discrete optimization, under the notion of Lovász' extension [13].

\footnotetext{
Keywords and phrases. Generalized coarea formula, total variation, anisotropic perimeter.

1 CMAP, École polytechnique, CNRS 91128, Palaiseau, France. antonin.chambolle@polytechnique.fr

2 Dipartimento di Matematica, Facoltà di Ingegneria, Università degli Studi di Brescia, Via Valotti 9, 25133 Brescia, Italy.

alessandro.giacomini@ing.unibs.it

3 Dipartimento di Matematica, I Facoltà di Ingegneria, Politecnico di Torino, c.so Duca degli Abruzzi 24, 10129 Torino, Italy. luca.lussardi@polito.it
} 
We will then study the continuous limit of our energies, as the discretization step $h$ goes to 0 (and the number of pixels/voxels in $\mathcal{V}$ to infinity), providing a very simple representation formula for the limit. In particular, it will be obvious from this formula that simple approximation procedures only provide "crystalline" energies, as already observed for instance in [3].

To be more specific, we consider in this paper an "interaction potential" $F:\{0,1\}^{\Sigma} \rightarrow[0,+\infty)$, which is a nonnegative function of binary vectors of $\{0,1\}^{\Sigma}$, where $\Sigma \subset \mathbb{Z}^{N}$ is a finite (small) set of "neighbors". We assume, in addition, that $F$ satisfies the submodularity condition

$$
F(u \wedge v)+F(u \vee v) \leq F(u)+F(v)
$$

for any $u, v \in\{0,1\}^{\Sigma}$, where $(u \wedge v)_{i}:=\min \left\{u_{i}, v_{i}\right\}$ and $(u \vee v)_{i}:=\max \left\{u_{i}, v_{i}\right\}$. Defining, for $x \in \mathbb{R}^{N}$ and $u$ a real-valued function, the vector $u[x+h \Sigma]=\left(u(x+h i)_{i \in \Sigma}\right) \in \mathbb{R}^{\Sigma}$, we will study the asymptotic behavior as $h \rightarrow 0$ of functionals of the type

$$
J_{h}(E, \Omega):=h^{N-1} \sum_{x \in I^{h}(\Omega) \cap h \mathbb{Z}^{N}} F\left(\chi_{E}[x+h \Sigma]\right),
$$

where here, $\Omega$ is a bounded open subset of $\mathbb{R}^{N}$ and $E$ is a discrete subset of the discrete lattice $\mathcal{V}=h \mathbb{Z}^{N} \cap \Omega$ ( $E$ is also identified to the union of the cubes $Q_{x}^{h}=x+\left[0, h\left[^{N}, x \in E\right.\right.$, and $\chi_{E}$ is its characteristic function). The notation $I^{h}(\Omega)$ stands for the points $x$ such that $x+h \Sigma \subset \Omega$, so that the sum in (1.2) involves only the nodes $x \in h \mathbb{Z}^{N}$ such that $x+h \Sigma \subset \Omega$.

The functional (1.2) is a sort of nonlocal anisotropic discrete perimeter of $E$. In fact it penalizes the boundary of $E$ in a nonlocal way, since an interface at the boundary with a vertex $x$ interacts with the behavior of $E$ on the cubes with vertices $x+h \Sigma$. The nonlocality vanishes as $h \rightarrow 0$ since its radius of action is given by $h \cdot \operatorname{diam}(\Sigma)$. The anisotropy is introduced by the function $F$, which can weight interfaces with various orientations in different ways.

The result of this paper concerns the asymptotic behavior of the discrete perimeters (1.2) as $h \rightarrow 0$ in the variational sense of $\Gamma$-convergence (see Sect. 2 for the definition) with respect to the $L^{1}$-topology on the family of discrete sets (that is $L^{1}$ convergence of characteristic functions). Under mild assumptions on $F$ and $\Omega$, we prove that (see Thm. 4.2) the discrete perimeters $\Gamma$-converge to the continuous anisotropic perimeter which for a sufficiently regular set $E$ (a set with Lipschitz boundary for instance) is given by

$$
J(E, \Omega)=\int_{\partial E} F\left(\nu_{E} \cdot \Sigma\right) d A
$$

where $\nu_{E}$ is the inner normal at the boundary and $\left(\nu_{E} \cdot \Sigma\right)=\left(\nu_{E} \cdot y\right)_{y \in \Sigma}$. This means that solutions of discrete minimization problems involving our discrete perimeters will be close, in the limit of the continuous setting, to minimizers of problems involving (1.3).

To be more precise, the class on which the $\Gamma$-limit is defined is given by the family of sets with finite perimeter in $\Omega[2,10]$. As a consequence, for a general set $E$, the boundary involved in the functional (1.3) is the reduced boundary $\partial^{*} E$, the inner normal $\nu_{E}$ is intended in a measure theoretical sense (see Sect. 2), and the area measure $d A$ has to be replaced by the $(N-1)$-dimensional Hausdorff measure $\mathcal{H}^{N-1}$. The function $F$ appearing in (1.3) is the extension to $\mathbb{R}^{\Sigma}$ of the submodular function $F$ by means of the formula

$$
F(u)=\int_{-\infty}^{+\infty} F\left(\chi_{\{u>s\}}\right) \mathrm{d} s
$$

where $\{u>s\}:=\{x \in \Sigma: u(x)>s\}$. Formula (1.4) is a coarea formula for the function $F$ since it relates the value $F(u)$ to the behavior of $F$ on the "boundary" of the level sets $\{u>s\}$ (compare with Eq. (2.3) which gives the classical coarea formula for functions of bounded variation). 
In view of the result on discrete perimeters, we obtain a $\Gamma$-convergence result for the functionals $J_{h}(\cdot, \Omega)$ extended to the class of piecewise constant functions $u$ relative to the grid $h \mathbb{Z}^{N}$. More precisely we consider $u$ of the form

$$
u=\sum_{x \in h \mathbb{Z}^{N}} a_{x} \chi_{Q_{x}^{h}}, \quad a_{x} \in \mathbb{R}
$$

and

$$
J_{h}(u, \Omega)=h^{N-1} \sum_{x \in I^{h}(\Omega) \cap h \mathbb{Z}^{N}} F(u[x+h \Sigma]) .
$$

As the functional (1.2) could be thought of as a discrete perimeter, the functional (1.5) could be considered as a sort of discrete total variation of the function $u$. Clearly it inherits the nonlocal and anisotropic features of the discrete perimeter. We show that the $\Gamma$-limit in the $L^{1}$-topology is given by the anisotropic total variation

$$
J(u, \Omega)=\int_{\Omega} F\left(\frac{D u}{|D u|} \cdot \Sigma\right) \mathrm{d}|D u|,
$$

where $u$ belongs to the space $B V(\Omega)$ of functions of bounded variation (see Sect. 2 ), $D u /|D u| \in \mathbb{S}^{N-1}$ denoting the Radon-Nikodym derivative of $D u$ with respect to its total variation $|D u|$. This $\Gamma$-convergence result is a simple consequence of the result on sets and of the fact that the functionals $J_{h}$ satisfy the generalized coarea formula

$$
J_{h}(u, \Omega)=\int_{-\infty}^{+\infty} J_{h}\left(\chi_{\{u>s\}}, \Omega\right) \mathrm{d} s,
$$

so that the behavior of $J_{h}$ on piecewise constant functions is completely determined by the discrete perimeters for sets. We infer the result from general properties of functionals on $L^{1}(\Omega)$ that satisfy a coarea formula like (1.7), which we study in Section 3. This class of functionals, denoted by $G C(\Omega)$, was investigated by Visintin $[16,17]$ in connection with phase transition problems. As a consequence of our $\Gamma$-convergence result, the discrete total variations (1.5) can be used to approximate Total Variation Minimization procedures in image denoising involving (1.6) (see Cor. 4.3).

The paper is organized as follows. Section 2 contains the notation employed in the paper, and some basic facts concerning sets with finite perimeter, functions of bounded variation and $\Gamma$-convergence. In Section 3 we consider the class $G C(\Omega)$ of functionals on $L^{1}(\Omega)$ which satisfy the generalized coarea formula: in particular we prove that $G C(\Omega)$ is closed under $\Gamma$-convergence, and that the limit can be recovered by the behavior on characteristic functions of Borel sets. Section 4 contains the main $\Gamma$-convergence result formulated for the discrete total variations (1.5). We exploit the reduction to the class of discrete sets, and Sections 4.1 and 4.2 contain the proof of the two inequalities characterizing the $\Gamma$-convergence of the discrete perimeters.

\section{NOTATION AND PRELIMINARIES}

Let $A$ be an open subset of $\mathbb{R}^{N}$. We will say that $A$ has a continuous boundary if $\partial A$ can be covered by finitely many balls $B$ such that, in each ball, $B \cap A$ is the subgraph of a continuous function (after an appropriate change of coordinates). If these functions are Lipschitz continuous, we say that $A$ has a Lipschitz boundary.

For any $p \in\left[1,+\infty\right.$ [ we will denote by $L^{p}(A)$ the usual space of all $p$-summable functions on $A$, and by $L^{\infty}(A)$ the space of measurable functions on $A$ which are essentially bounded. Given $u, v \in L^{1}(A)$, we set

$$
u \wedge v:=\min \{u, v\} \quad \text { and } \quad u \vee v:=\max \{u, v\}
$$

In the following, we recall some basic facts concerning function of bounded variation and sets with finite perimeter which we need in the following sections, together with some basic definitions and results concerning Г-convergence.

Functions of bounded variation and sets with finite perimeter. For an exhaustive treatment of the subject, we refer the reader to [2]. 
We say that $u$ has bounded variation in $A$ and we write $u \in B V(A)$ if $u \in L^{1}(A)$ and

$$
|D u|(A)=\sup \left\{\int_{A} u \operatorname{div} \varphi \mathrm{d} x: \varphi \in C_{c}^{1}(A),\|\varphi\|_{\infty} \leq 1\right\}<+\infty .
$$

$|D u|(A)$ is referred to as the total variation of $u$.

If $E \subseteq A$ is a Borel set, we say that $E$ has finite perimeter in $A$ if $\chi_{E} \in B V(A)$, and we set

$$
\operatorname{Per}(E, A):=\left|D \chi_{E}\right|(A) .
$$

$\operatorname{Per}(E, A)$ is called the perimeter of $E$ in $A$. It turns out that

$$
\operatorname{Per}(E, A)=\mathcal{H}^{N-1}\left(\partial^{*} E \cap A\right) \text {, and } D \chi_{E}=\nu_{E} \mathcal{H}^{N-1}\left\llcorner\partial^{*} E,\right.
$$

where $\partial^{*} E$ denotes the reduced boundary of $E$, which, up to a $\mathcal{H}^{N-1}$-negligible set, coincides with the (larger) set of points $x$ such that there exists a unit vector $\nu_{E}(x)$ with

$$
\frac{E-x}{\varrho} \rightarrow\left\{y \in \mathbb{R}^{N}: y \cdot \nu_{E}(x)>0\right\} \quad \text { as } \varrho \rightarrow 0 \text { in } L_{\mathrm{loc}}^{1}\left(\mathbb{R}^{N}\right) .
$$

The unitary vector $\nu_{E}(x)$ is usually referred to as the interior normal to $E$ at $x$. $\mathcal{H}^{N-1}$ denotes the $(N-1)$ dimensional Hausdorff measure, which is a generalization to arbitrary sets of the usual $(N-1)$-area measure. The points of $\partial^{*} E$ are also called regular points of $\partial E$.

If $u \in B V(A)$, the following coarea formula holds:

$$
|D u|(A)=\int_{-\infty}^{+\infty} \operatorname{Per}(\{x \in A: u(x)>s\}, A) \mathrm{d} s=\int_{-\infty}^{+\infty}\left|D \chi_{\{u>s\}}\right|(A) \mathrm{d} s .
$$

Finally we recall the following compactness result (which is a variant of Rellich's theorem). If $A$ is bounded and with Lipschitz boundary, and $\left(u_{n}\right)_{n \in \mathbb{N}}$ is a sequence in $B V(A)$ such that $\left\|u_{n}\right\|_{L^{1}(A)}+\left|D u_{n}\right|(A)$ is bounded, then there exist a subsequence $\left(u_{n_{k}}\right)_{k \in \mathbb{N}}$ and a function $u \in B V(A)$ such that

$$
u_{n_{k}} \rightarrow u \quad \text { in } L^{1}(A)
$$

and

$$
|D u|(A) \leq \liminf _{k \rightarrow \infty}\left|D u_{n_{k}}\right|(A)
$$

$\Gamma$-convergence. Let us recall the definition and some basic properties of De Giorgi's $\Gamma$-convergence in metric spaces. We refer the reader to $[5,8]$ for an exhaustive treatment of this subject. Let $(X, d)$ be a metric space. We say that a sequence $F_{n}: X \rightarrow[-\infty,+\infty] \Gamma$-converges to $F: X \rightarrow[-\infty,+\infty]($ as $n \rightarrow \infty)$ if for all $u \in X$ we have

(i) ( $\Gamma$-lim inf inequality) for every sequence $\left(u_{n}\right)_{n \in \mathbb{N}}$ converging to $u$ in $X$,

$$
\liminf _{n \rightarrow \infty} F_{n}\left(u_{n}\right) \geq F(u)
$$

(ii) ( $\Gamma$-lim sup inequality) there exists a sequence $\left(u_{n}\right)_{n \in \mathbb{N}}$ converging to $u$ in $X$, such that

$$
\limsup _{n \rightarrow \infty} F_{n}\left(u_{n}\right) \leq F(u) .
$$


The function $F$ is called the $\Gamma$-limit of $\left(F_{n}\right)_{n \in \mathbb{N}}$ (with respect to $d$ ). Given a family $\left(F_{h}\right)_{h>0}$ of functionals on $X$, we say that $F_{h} \Gamma$-converges to $F$ as $h \rightarrow 0$ if for every sequence $h_{n} \rightarrow 0$ we have that $F_{h_{n}} \Gamma$-converges to $F$ as $n \rightarrow \infty$.

$\Gamma$-convergence is a convergence of variational type as explained in the following proposition.

Proposition 2.1. Assume that the family $\left(F_{h}\right)_{h>0} \Gamma$-converges to $F$ and that there exists a compact set $K \subseteq X$ such that for all $h>0$

$$
\inf _{u \in K} F_{h}(u)=\inf _{u \in X} F_{h}(u) .
$$

Then $F$ admits a minimum on $X, \inf _{X} F_{h} \rightarrow \min _{X} F$ as $h \rightarrow 0$, and any limit point of any sequence $\left(u_{h}\right)_{h>0}$ such that

$$
\lim _{h \rightarrow 0}\left(F_{h}\left(u_{h}\right)-\inf _{u \in X} F_{h}(u)\right)=0
$$

is a minimizer of $F$.

\section{Generalized COAREA Formula}

In the following, let $\Omega \subset \mathbb{R}^{N}$ be an open and bounded set.

Definition 3.1. Let $J: L^{1}(\Omega) \rightarrow[0,+\infty]$ be a proper functional. We say that $J$ satisfies the generalized coarea formula if for every $u \in L^{1}(\Omega)$

$$
J(u)=\int_{-\infty}^{+\infty} J\left(\chi_{\{u>s\}}\right) \mathrm{d} s
$$

with the convention $J(u)=+\infty$ if the map $s \mapsto J\left(\chi_{\{u>s\}}\right)$ is not measurable. We denote by $G C(\Omega)$ the class of functionals satisfying (3.1).

The class $G C(\Omega)$ has been introduced by Visintin [16] and investigated, in the discrete case, by Chambolle and Darbon [6]. In a slightly different setting, the formula (3.1) is a variant of the extension introduced by Lovász in [13] and well-known in combinatorial and linear optimization.

An example of functional satisfying (3.1) is given by the total variation (2.2) in view of the coarea formula (2.3). Other examples are treated in [17]:

$$
J(u)=\int_{\Omega \times \Omega}|u(x)-u(y)||x-y|^{-(N+r)} \mathrm{d} x \mathrm{~d} y, \quad \forall r \in(0,1)
$$

and

$$
J(u)=\int_{\Omega \times \mathbb{R}^{+}}\left(\underset{B_{h}(x) \cap \Omega}{\operatorname{ess} \sup } u-\underset{B_{h}(x) \cap \Omega}{\operatorname{ess} \inf } u\right) h^{-(1+r)} \mathrm{d} x \mathrm{~d} h, \quad \forall r \in(0,1) .
$$

The next proposition contains some elementary consequences of formula (3.1).

Proposition 3.2. Let $J \in G C(\Omega)$. Then for every $u \in L^{1}(\Omega)$ the following facts hold:

(i) $J(\lambda u)=\lambda J(u)$ for every $\lambda>0$;

(ii) $J(u+c)=J(u)$ for every $c \in \mathbb{R}$;

(iii) $J(c)=0$ for every $c \in \mathbb{R}$.

Moreover if $J$ is convex, for every $u, v \in L^{1}(\Omega)$ we have

(iv) $J(u \wedge v)+J(u \vee v) \leq J(u)+J(v)$.

Proof. Let $\lambda>0, u \in L^{1}(\Omega)$ and $c \in \mathbb{R}$. Then

$$
J(\lambda u)=\int_{-\infty}^{+\infty} J\left(\chi_{\left\{u>\frac{s}{\lambda}\right\}}\right) \mathrm{d} s=\lambda \int_{-\infty}^{+\infty} J\left(\chi_{\{u>t\}}\right) \mathrm{d} t=\lambda J(u)
$$


and

$$
J(u+c)=\int_{-\infty}^{+\infty} J\left(\chi_{\{u>s-c\}}\right) \mathrm{d} s=\int_{-\infty}^{+\infty} J\left(\chi_{\{u>t\}}\right) \mathrm{d} t=J(u)
$$

so that (i) and (ii) follow.

Let us prove (iii). In view of (ii), it suffices to show that $J(0)=0$. Suppose by contradiction that $J(0)>0$. Then for every $u \in L^{\infty}(\Omega)$ we have

$$
J(u)=\int_{-\infty}^{+\infty} J\left(\chi_{\{u>s\}}\right) \mathrm{d} s \geq \int_{\substack{\operatorname{ess} \sup u \\ \Omega}}^{+\infty} J(0) \mathrm{d} s=+\infty .
$$

By the generalized coarea formula, we deduce that $J(u)=+\infty$ for every $u \in L^{1}(\Omega)$. But this is against the fact that $J$ is proper, so that point (iii) is proved.

Let us show (iv). Since we have

$$
\begin{aligned}
J\left(\chi_{\{u>s\}} \wedge \chi_{\{v>s\}}\right)+J\left(\chi_{\{u>s\}} \vee \chi_{\{v>s\}}\right) & =\int_{0}^{2} J\left(\chi_{\left\{\chi_{\{u>s\}} \wedge \chi_{\{v>s\}}+\chi_{\{u>s\}} \vee \chi_{\{v>s\}}>t\right\}}\right) \mathrm{d} t \\
& =\int_{-\infty}^{+\infty} J\left(\chi_{\left\{\chi_{\{u>s\}} \wedge \chi_{\{v>s\}}+\chi_{\{u>s\}} \vee \chi_{\{v>s\}}>t\right\}}\right) \mathrm{d} t
\end{aligned}
$$

by the generalized coarea formula (3.1) we get

$$
J\left(\chi_{\{u>s\}} \wedge \chi_{\{v>s\}}\right)+J\left(\chi_{\{u>s\}} \vee \chi_{\{v>s\}}\right)=J\left(\chi_{\{u>s\}} \wedge \chi_{\{v>s\}}+\chi_{\{u>s\}} \vee \chi_{\{v>s\}}\right)=J\left(\chi_{\{u>s\}}+\chi_{\{v>s\}}\right) .
$$

Notice that if $J$ is convex, by point (i) we deduce that $J$ is subadditive. Then we obtain

$$
J\left(\chi_{\{u>s\}} \wedge \chi_{\{v>s\}}\right)+J\left(\chi_{\{u>s\}} \vee \chi_{\{v>s\}}\right) \leq J\left(\chi_{\{u>s\}}\right)+J\left(\chi_{\{v>s\}}\right) .
$$

Observe that for any $s \in \mathbb{R}$ we have $\{u \wedge v>s\}=\{u>s\} \cap\{v>s\}$ and $\{u \vee v>s\}=\{u>s\} \cup\{v>s\}$ so that

We conclude by (3.3)

$$
\chi_{\{u \wedge v>s\}}=\chi_{\{u>s\}} \wedge \chi_{\{v>s\}} \quad \text { and } \quad \chi_{\{u \vee v>s\}}=\chi_{\{u>s\}} \vee \chi_{\{v>s\}} .
$$

$$
\begin{aligned}
J(u \wedge v)+J(u \vee v) & =\int_{-\infty}^{+\infty}\left[J\left(\chi_{\{u \wedge v>s\}}\right)+J\left(\chi_{\{u \vee v>s\}}\right)\right] \mathrm{d} s \\
& =\int_{-\infty}^{+\infty}\left[J\left(\chi_{\{u>s\}} \wedge \chi_{\{v>s\}}\right)+J\left(\chi_{\{u>s\}} \vee \chi_{\{v>s\}}\right)\right] \mathrm{d} s \\
& \leq \int_{-\infty}^{+\infty} J\left(\chi_{\{u>s\}}\right) \mathrm{d} s+\int_{-\infty}^{+\infty} J\left(\chi_{\{v>s\}}\right) \mathrm{d} s=J(u)+J(v)
\end{aligned}
$$

so that (iv) follows and the proof is complete.

We will need the following lemma concerning the approximation of Lebesgue integral by means of Riemann sums.

Lemma 3.3. Let $\left.f \in L^{1}(\mathbb{R}), t \in\right] 0,1[$ and let us set

$$
s_{n}(t):=\frac{1}{n} \sum_{k \in \mathbb{Z}} f\left(\frac{k+t}{n}\right) .
$$


Then up to a subsequence we have

$$
\left.\lim _{n \rightarrow \infty} s_{n}(t)=\int_{-\infty}^{+\infty} f(\tau) \mathrm{d} \tau \quad \text { for a.e. } t \in\right] 0,1[\text {. }
$$

Proof. For any $n \in \mathbb{N}$ we easily get

$$
\int_{-\infty}^{+\infty} f(\tau) \mathrm{d} \tau=\frac{1}{n} \sum_{k \in \mathbb{Z}} \int_{0}^{1} f\left(\frac{k+r}{n}\right) \mathrm{d} r
$$

Then for $t \in] 0,1[$ we have

$$
\begin{array}{r}
\int_{0}^{1}\left|\int_{-\infty}^{+\infty} f(\tau) \mathrm{d} \tau-s_{n}(t)\right| \mathrm{d} t=\int_{0}^{1}\left|\int_{-\infty}^{+\infty} f(\tau) \mathrm{d} \tau-\frac{1}{n} \sum_{k \in \mathbb{Z}} f\left(\frac{k+t}{n}\right)\right| \mathrm{d} t \\
=\int_{0}^{1}\left|\frac{1}{n} \sum_{k \in \mathbb{Z}} \int_{0}^{1} f\left(\frac{k+r}{n}\right) \mathrm{d} r-\frac{1}{n} \sum_{k \in \mathbb{Z}} f\left(\frac{k+t}{n}\right)\right| \mathrm{d} t \\
\leq \frac{1}{n} \sum_{k \in \mathbb{Z}} \int_{0}^{1} \int_{0}^{1}\left|f\left(\frac{k+r}{n}\right)-f\left(\frac{k+t}{n}\right)\right| \mathrm{d} r \mathrm{~d} t .
\end{array}
$$

But

$$
\begin{aligned}
& \frac{1}{n} \sum_{k \in \mathbb{Z}} \int_{0}^{1} \int_{0}^{1}\left|f\left(\frac{k+r}{n}\right)-f\left(\frac{k+t}{n}\right)\right| \mathrm{d} r \mathrm{~d} t \\
& \leq \frac{1}{n} \sum_{k \in \mathbb{Z}} \int_{0}^{1} \int_{0}^{1}\left[\left|f\left(\frac{k+r}{n}\right)-f\left(\frac{k+r+t}{n}\right)\right|+\left|f\left(\frac{k+r+t}{n}\right)-f\left(\frac{k+t}{n}\right)\right|\right] \mathrm{d} r \mathrm{~d} t \\
& =\int_{0}^{1}\left\|f(\cdot)-f\left(\cdot+\frac{t}{n}\right)\right\|_{L^{1}(\mathbb{R})} \mathrm{d} t+\int_{0}^{1}\left\|f(\cdot)-f\left(\cdot+\frac{r}{n}\right)\right\|_{L^{1}(\mathbb{R})} \mathrm{d} r .
\end{aligned}
$$

The last terms tend to zero by continuity of the translation operator in $L^{1}(\mathbb{R})$. We conclude that

$$
s_{n} \rightarrow \int_{-\infty}^{+\infty} f(\tau) \mathrm{d} \tau \quad \text { in } L^{1}(0,1)
$$

so that, up to a subsequence, pointwise almost everywhere convergence follows.

In view of (3.1), functionals in the class $G C(\Omega)$ are completely determined by their behavior on characteristic functions of Borel sets contained in $\Omega$. The next result gives a sufficient condition for the convexity of lower semicontinuous functionals in $G C(\Omega)$ in terms of the submodularity property (iv) of the previous proposition only on characteristic functions.

Proposition 3.4. Let $J \in G C(\Omega)$ be a lower semicontinuous functional such that

$$
J\left(\chi_{E \cap E^{\prime}}\right)+J\left(\chi_{E \cup E^{\prime}}\right) \leq J\left(\chi_{E}\right)+J\left(\chi_{E^{\prime}}\right)
$$

for every pair of Borel sets $E, E^{\prime}$ in $\Omega$. Then $J$ is convex. 
Proof. Since by Proposition $3.2 \mathrm{~J}$ is positively one-homogeneous, it is sufficient to show that

$$
J(u+v) \leq J(u)+J(v)
$$

for any $u, v \in L^{1}(\Omega)$.

We claim that the following representation formula holds for every function $u$ which is positive, bounded and with integer values:

$$
J(u)=\min \left\{\sum_{i=1}^{m} J\left(\chi_{E_{i}}\right): m \geq 1, u=\sum_{i=1}^{m} \chi_{E_{i}}\right\} .
$$

In order to prove (3.5), we can clearly assume that $J(u)<+\infty$ and $J(v)<+\infty$. Hence by (3.1) the maps $s \mapsto J\left(\chi_{\{u>s\}}\right)$ and $s \mapsto J\left(\chi_{\{v>s\}}\right)$ belong to $L^{1}(\mathbb{R})$.

Firstly let us assume $0 \leq u \leq 1$ and $0 \leq v \leq 1$. For every $n \in \mathbb{N}, n>0$, let us set for $t \in] 0,1[$

$$
u_{n}:=\frac{1}{n} \sum_{k \in \mathbb{N}} \chi_{\left\{u>\frac{k+t}{n}\right\}} \quad \text { and } \quad v_{n}:=\frac{1}{n} \sum_{k \in \mathbb{N}} \chi_{\left\{v>\frac{k+t}{n}\right\}}
$$

By applying Lemma 3.3, we can choose $t \in] 0,1[$ in such a way that

$$
\lim _{n \rightarrow \infty} \frac{1}{n} \sum_{k \in \mathbb{N}} J\left(\chi_{\left\{u>\frac{k+t}{n}\right\}}\right)=\int_{0}^{1} J\left(\chi_{\{u>s\}}\right) \mathrm{d} s
$$

and

$$
\lim _{n \rightarrow \infty} \frac{1}{n} \sum_{k \in \mathbb{N}} J\left(\chi_{\left\{v>\frac{k+t}{n}\right\}}\right)=\int_{0}^{1} J\left(\chi_{\{v>s\}}\right) \mathrm{d} s .
$$

By construction $u_{n} \rightarrow u$ and $v_{n} \rightarrow v$ in $L^{1}(\Omega)$. Then by positive homogeneity, and assuming the representation formula (3.6) holds, we get

$$
J\left(u_{n}+v_{n}\right)=J\left(\frac{1}{n} \sum_{k \in \mathbb{N}} \chi_{\left\{u>\frac{k+t}{n}\right\}}+\chi_{\left\{v>\frac{k+t}{n}\right\}}\right) \leq \frac{1}{n} \sum_{k \in \mathbb{N}} J\left(\chi_{\left\{u>\frac{k+t}{n}\right\}}\right)+J\left(\chi_{\left\{v>\frac{k+t}{n}\right\}}\right) .
$$

The right-hand side converges by construction to $J(u)+J(v)$, and thus, by lower semicontinuity of $J$, we have that (3.5) follows.

In the case $m \leq u \leq M$ and $m \leq v \leq M$, one can easily show again that (3.5) holds by considering the functions $(u-m) /(M-m)$ and $(v-m) /(M-m)$, and taking into account the general properties of $J$.

Finally, for $u, v \in L^{1}(\Omega)$ and for $T>0$, let us consider $u_{T}:=-T \vee u \wedge T$ and $v_{T}:=-T \vee v \wedge T$. Since $u_{T} \rightarrow u$ and $v_{T} \rightarrow v$ in $L^{1}(\Omega)$, by the lower semicontinuity of $J$ we obtain

$$
\begin{aligned}
& J(u+v) \leq \liminf _{T \rightarrow+\infty} J\left(u_{T}+v_{T}\right) \leq \liminf _{T \rightarrow+\infty}\left(J\left(u_{T}\right)+J\left(v_{T}\right)\right) \leq \limsup _{T \rightarrow+\infty} J\left(u_{T}\right)+\limsup _{T \rightarrow+\infty} J\left(v_{T}\right) \\
& \quad=\lim _{T \rightarrow+\infty} \int_{-T}^{T} J\left(\chi_{\{u>s\}}\right) \mathrm{d} s+\lim _{T \rightarrow+\infty} \int_{-T}^{T} J\left(\chi_{\{v>s\}}\right) \mathrm{d} s=J(u)+J(v),
\end{aligned}
$$

so that (3.5) follows.

In order to conclude the proof, we have to check claim (3.6). Let $M:=\max u$. Since $u$ is positive and integer valued, we can write $u=\sum_{i=1}^{M} \chi_{\{u \geq i\}}$. For any $i \in\{1, \ldots, M\}$ we have

$$
\int_{i-1}^{i} J\left(\chi_{\{u>s\}}\right) \mathrm{d} s=J\left(\chi_{\{u \geq i\}}\right)
$$


so that

$$
J(u)=\int_{0}^{+\infty} J\left(\chi_{\{u>s\}}\right) \mathrm{d} s=\sum_{i=1}^{M} J\left(\chi_{\{u \geq i\}}\right) .
$$

Hence

$$
J(u) \geq \inf \left\{\sum_{i=1}^{m} J\left(\chi_{E_{i}}\right): m \geq 1, u=\sum_{i=1}^{m} \chi_{E_{i}}\right\} .
$$

In order to prove the opposite inequality let $u=\sum_{i=1}^{m} \chi_{E_{i}}$ for some Borel set $E_{i} \subseteq \Omega$ and $m \geq 1$. Observe that for any $r, s \in\{1, \ldots, m\}$ with $r \neq s$ we also have

$$
u=\chi_{E_{r} \cap E_{s}}+\chi_{E_{r} \cup E_{s}}+\sum_{\substack{i \neq r \\ i \neq s}} \chi_{E_{i}} .
$$

From (3.4) we get

$$
J\left(\chi_{E_{r} \cap E_{s}}\right)+J\left(\chi_{E_{r} \cup E_{s}}\right)+\sum_{\substack{i \neq r \\ i \neq s}} J\left(\chi_{E_{i}}\right) \leq \sum_{i=1}^{m} J\left(\chi_{E_{i}}\right) .
$$

Then by induction it is easy to see that

$$
\begin{array}{r}
\inf \left\{\sum_{i=1}^{m} J\left(\chi_{E_{i}}\right): m \geq 1, u=\sum_{i=1}^{m} \chi_{E_{i}}\right\} \geq \inf \left\{\sum_{i=1}^{m} J\left(\chi_{E_{i}}\right): m \geq 1, u=\sum_{i=1}^{m} \chi_{E_{i}}, E_{1} \supseteq E_{2} \supseteq \cdots \supseteq E_{m}\right\} \\
=\sum_{i=1}^{M} J\left(\chi_{\{u \geq i\}}\right)=J(u) .
\end{array}
$$

Hence claim (3.6) holds true, so that the proof is concluded.

The following proposition deals with the stability of the class $G C(\Omega)$ with respect to the $\Gamma$-convergence.

Proposition 3.5. Let $\left(J_{n}\right)_{n \in \mathbb{N}}$ be a sequence of convex functionals in $G C(\Omega)$ such that there exists a functional $\tilde{J}$ defined on characteristic functions of Borel sets which satisfies the following conditions:

(a) for every Borel set $E \subseteq \Omega$ and for every sequence of Borel sets $\left(E_{n}\right)_{n \in \mathbb{N}}$ contained in $\Omega$ such that $\chi_{E_{n}} \rightarrow \chi_{E}$ in $L^{1}(\Omega)$ we have

$$
\tilde{J}\left(\chi_{E}\right) \leq \liminf _{n \rightarrow \infty} J_{n}\left(\chi_{E_{n}}\right) ;
$$

(b) for every Borel set $E \subseteq \Omega$ there exists a sequence of Borel sets $\left(E_{n}\right)_{n \in \mathbb{N}}$ contained in $\Omega$ with $\chi_{E_{n}} \rightarrow \chi_{E}$ in $L^{1}(\Omega)$ and such that

$$
\limsup _{n \rightarrow \infty} J_{n}\left(\chi_{E_{n}}\right) \leq \tilde{J}\left(\chi_{E}\right)
$$

Then setting

$$
J(u):=\int_{-\infty}^{+\infty} \tilde{J}\left(\chi_{\{u>s\}}\right) \mathrm{d} s,
$$

we have $J \in G C(\Omega)$ and the sequence $\left(J_{n}\right)_{n \in \mathbb{N}} \Gamma$-converges to $J$ in the $L^{1}$-topology.

Conversely let $\left(J_{n}\right)_{n \in \mathbb{N}}$ be a sequence of functionals in $G C(\Omega)$ which $\Gamma$-converges to a proper functional $J: L^{1}(\Omega) \rightarrow[0,+\infty]$. Then $J \in G C(\Omega)$ and its restriction $\tilde{J}$ to the family of characteristic functions of Borel subsets of $\Omega$ satisfies conditions (a) and (b). 
Remark 3.6. Notice that it follows that for convex functionals in $G C(\Omega)$, the $\Gamma$-convergence is equivalent to the $\Gamma$-convergence on the corresponding (submodular) set functions, that is, the restriction to characteristic functions of the original functionals. However, the last statement in Proposition 3.5 is also true without assuming any convexity of the functions $J_{n}$. Notice that there exist functionals in $G C(\Omega)$ which are lower semicontinuous but not convex, so that convexity cannot be gained by relaxation. (It suffices to consider functionals of the form (4.4) with $\Omega$ and $h$ chosen in such a way that the summation involves only one square, and the function $F$ is not submodular on binary vectors.)

Proof of Proposition 3.5. Notice that $\tilde{J}$ is, by construction, lower semicontinuous on characteristic functions, so that the map $s \mapsto \tilde{J}\left(\chi_{\{u>s\}}\right)$ is measurable for every $u \in L^{1}(\Omega)$. Hence the definition of $J$ is well posed.

In order to prove the $\Gamma$-convergence result, we need to check $\Gamma$-lim inf and $\Gamma$-lim sup inequalities (see Sect. 2). Let us start with the $\Gamma$-lim inf inequality. Let $u_{n} \rightarrow u$ in $L^{1}(\Omega)$. Up to a subsequence, we can assume that $\chi_{\left\{u_{n}>s\right\}} \rightarrow \chi_{\{u>s\}}$ in $L^{1}(\Omega)$ for a.e. $s \in \mathbb{R}$. By Fatou's Lemma, the generalized coarea formula (3.1) and assumption (a) we get

$$
\liminf _{n \rightarrow \infty} J_{n}\left(u_{n}\right) \geq \int_{-\infty}^{+\infty} \liminf _{n \rightarrow \infty} J_{n}\left(\chi_{\left\{u_{n}>s\right\}}\right) \mathrm{d} s \geq \int_{-\infty}^{+\infty} \tilde{J}\left(\chi_{\{u>s\}}\right) \mathrm{d} s=J(u)
$$

so that the $\Gamma$-lim inf inequality follows.

Let us come to the $\Gamma$-lim sup inequality. We can clearly assume that the map $s \mapsto \tilde{J}\left(\chi_{\{u>s\}}\right)$ belongs to $L^{1}(\mathbb{R})$. Notice that the subspace given by (finite) linear combinations of characteristic functions is dense with respect to the energy $J$. In fact, if $0 \leq u \leq 1$, by Lemma 3.3 we can choose $t \in] 0,1[$ such that

$$
\frac{1}{m} \sum_{k \in \mathbb{N}} \chi_{\left\{u>\frac{k+t}{m}\right\}} \rightarrow u \quad \text { in } L^{1}(\Omega)
$$

and $($ since $\tilde{J}(0)=\tilde{J}(1)=0)$

$$
\lim _{m \rightarrow \infty} \frac{1}{m} \sum_{k \in \mathbb{N}} \tilde{J}\left(\chi_{\left\{u>\frac{k+t}{m}\right\}}\right)=\int_{0}^{1} \tilde{J}\left(\chi_{\{u>s\}}\right) \mathrm{d} s=\int_{-\infty}^{+\infty} \tilde{J}\left(\chi_{\{u>s\}}\right) \mathrm{d} s .
$$

The case $m \leq u \leq M$ follows considering the function $(u-m) /(M-m)$. Finally, for $u \in L^{1}(\Omega)$, let us set $u_{T}:=-T \vee u \wedge T \rightarrow u$ for every $T>0$. Since $u_{T} \rightarrow u$ in $L^{1}(\Omega)$ and $J\left(u_{T}\right) \rightarrow J(u)$ as $T \rightarrow+\infty$, the density in energy follows by a diagonal argument. By general results of $\Gamma$-convergence, it suffices to prove the $\Gamma$-lim sup inequality for $u$ equal to a linear combination of characteristic functions. Since $J$ is invariant under translation, it is not restrictive to assume that $u=\sum_{k=1}^{m} a_{k} \chi_{E_{k}}$ with $a_{k} \geq 0$ for every $k=1, \ldots, m$, and $E_{m} \subseteq E_{m-1} \subseteq \cdots \subseteq E_{1}$. In this way we have

$$
J(u)=\sum_{k=1}^{m} a_{k} \tilde{J}\left(\chi_{E_{k}}\right)
$$

By condition (b), we can find Borel sets $E_{k}^{n}$ such that

$$
\chi_{E_{k}^{n}} \rightarrow \chi_{E_{k}} \quad \text { in } L^{1}(\Omega)
$$

as $n \rightarrow \infty$, and

$$
\limsup _{n \rightarrow \infty} J_{n}\left(\chi_{E_{k}^{n}}\right) \leq \tilde{J}\left(\chi_{E_{k}}\right) .
$$


Setting $u_{n}:=\sum_{k=1}^{m} a_{k} \chi_{E_{k}^{n}}$, we have $u_{n} \rightarrow u$ in $L^{1}(\Omega)$. Since $J_{n}$ is convex and positively one-homogeneous, and hence subadditive, we deduce

$$
\limsup _{n \rightarrow \infty} J_{n}\left(u_{n}\right) \leq \sum_{k=1}^{m} a_{k} \limsup _{n \rightarrow \infty} J_{n}\left(\chi_{E_{k}^{n}}\right) \leq \sum_{k=1}^{m} a_{k} \tilde{J}\left(\chi_{E_{k}}\right)=J(u),
$$

so that the $\Gamma$-lim sup inequality is proved.

Finally, the fact that $J \in G C(\Omega)$ follows since $J$ and $\tilde{J}$ coincide on characteristic functions. The proof of the first part of the proposition is thus complete.

Let us come to the second part. Clearly $\tilde{J}$ satisfies condition (a). In order to prove condition (b), let $E$ be a Borel subset of $\Omega$, and let $u_{n} \in L^{1}(\Omega)$ be such that $u_{n} \rightarrow \chi_{E}$ in $L^{1}(\Omega)$ and $\lim \sup _{n \rightarrow \infty} J_{n}\left(u_{n}\right) \leq \tilde{J}\left(\chi_{E}\right)$. Since for any $\delta \in(0,1)$

$$
J_{n}\left(u_{n}\right) \geq \int_{\delta}^{1-\delta} J_{n}\left(\chi_{\left\{u_{n}>s\right\}}\right) \mathrm{d} s
$$

there exists $s_{n} \in(\delta, 1-\delta)$ such that

$$
J_{n}\left(\chi_{\left\{u_{n}>s_{n}\right\}}\right) \leq \frac{1}{1-2 \delta} J_{n}\left(u_{n}\right)
$$

Let us set $E_{n}^{\delta}:=\chi_{\left\{u_{n}>s_{n}\right\}}$. We have clearly that $\chi_{E_{n}^{\delta}} \rightarrow \chi_{E}$ in $L^{1}(\Omega)$ and by (3.7) we deduce

$$
\limsup _{n \rightarrow \infty} J_{n}\left(\chi_{E_{n}^{\delta}}\right) \leq \frac{1}{1-2 \delta} \limsup _{n \rightarrow \infty} J_{n}\left(u_{n}\right) \leq \frac{1}{1-2 \delta} \tilde{J}\left(\chi_{E}\right) .
$$

Let us choose now $\delta=1 / m$. There exists $n_{m}$ such that for every $n \geq n_{m}$ we have

$$
\left\|\chi_{E_{n}^{1 / m}}-\chi_{E}\right\|_{L^{1}} \leq 1 / m
$$

and

$$
J_{n}\left(\chi_{E_{n}^{1 / m}}\right) \leq \frac{1}{1-2 / m} \tilde{J}\left(\chi_{E}\right)
$$

Moreover we may assume that $n_{m} \uparrow \infty$. If we set $E_{n}:=E_{n}^{1 / m}$ for $n_{m} \leq n<n_{m+1}$, we have that $\left(E_{n}\right)_{n \in \mathbb{N}}$ is the recovering sequence for which the $\Gamma$-lim sup inequality holds.

Finally, the fact that $J \in G C(\Omega)$ follows now from the first part of the proposition, and this concludes the proof.

\section{Discrete approximation OF ANisotropic tOtAl VARIATION}

Let $N \geq 1$ and $\Sigma \subset \mathbb{Z}^{N}$ be a finite set, and let $F:\{0,1\}^{\Sigma} \rightarrow[0,+\infty)$ be a nonnegative submodular function, i.e. $F(u \wedge v)+F(u \vee v) \leq F(u)+F(v)$ for any $u, v \in\{0,1\}^{\Sigma}$, with $F(0)=F\left(\chi_{\Sigma}\right)=0$. We extend $F$ to all vectors $u \in \mathbb{R}^{\Sigma}$ into a convex function by letting (see Prop. 3.4)

$$
F(u)=\int_{-\infty}^{+\infty} F\left(\chi_{\{u>s\}}\right) \mathrm{d} s
$$

where $\{u>s\}:=\{x \in \Sigma: u(x)>s\}$. We let

$$
\rho_{\Sigma}:=\max _{x \in \Sigma}|x|
$$


and we assume, in addition, the following coercivity assumption:

(A) $\Sigma$ contains 0 and the canonical basis $\left(e_{i}\right)_{i=1}^{N}$ of $\mathbb{R}^{N}$, and there exists $c>0$ such that for any $u \in \mathbb{R}^{\Sigma}$,

$$
F(u) \geq c \sum_{i=1}^{N}\left|u\left(e_{i}\right)-u(0)\right| .
$$

Notice that (4.1) is a discrete version of the generalized coarea formula (3.1).

Given $h>0$ and $x \in h \mathbb{Z}^{N}$ let us set

$$
Q_{x}^{h}:=x+h\left[0,1\left[^{N} .\right.\right.
$$

Let $V_{h}$ denote the space of functions $u: \mathbb{R}^{N} \rightarrow \mathbb{R}$ such that

$$
u=\sum_{x \in h \mathbb{Z}^{N}} u(x) \chi_{Q_{x}^{h}} .
$$

Notice that we have $V_{h} \subseteq L_{\text {loc }}^{1}\left(\mathbb{R}^{N}\right)$.

Let $\Omega \subset \mathbb{R}^{N}$ be an open and bounded set. We denote by $V_{h}(\Omega)$ the restriction to $\Omega$ of the functions in $V_{h}$. Let moreover $I^{h}(\Omega)$ denote the set of $x \in \mathbb{R}^{N}$ such that $x+h \Sigma \subset \Omega$. We consider the functional $J_{h}(\cdot, \Omega): L^{1}(\Omega) \rightarrow[0,+\infty[$ defined as

$$
J_{h}(u, \Omega):= \begin{cases}h^{N-1} \sum_{x \in I^{h}(\Omega) \cap h \mathbb{Z}^{N}} F(u[x+h \Sigma]) & \text { if } u \in V_{h}(\Omega) \\ +\infty & \text { if } u \in L^{1}(\Omega) \backslash V_{h}(\Omega),\end{cases}
$$

where for any $x \in I^{h}(\Omega), u[x+h \Sigma]$ is the vector $\left(u(x+h y)_{y \in \Sigma}\right)$ of $\mathbb{R}^{\Sigma}$.

The aim of this section is to study the asymptotic behavior of the functionals $J_{h}(\cdot, \Omega)$ as the size mesh $h$ vanishes: it is expected that they approximate some anisotropic total variation. The following proposition shows that the functionals $J_{h}(\cdot, \Omega)$ satisfy the generalized coarea formula (3.1).

Proposition 4.1. The functional $J_{h}(\cdot, \Omega)$ is convex and belongs to $G C(\Omega)$. Moreover, there exist $C_{2}>C_{1}>0$ such that for any open sets $A, B$ with $A \subset \subset B \subset \subset \Omega$, and for any $u \in V_{h}(\Omega)$, we have, if $h$ is small enough,

$$
C_{1}|D u|(A) \leq J_{h}(u, B) \leq C_{2}|D u|(\Omega) .
$$

Proof. From (4.1), we get that also $J_{h}$ satisfies (3.1). The submodularity of $F$ yields (3.4), hence $J_{h}$ is convex.

To show the estimate (4.5), it is enough to assume that $u \in V_{h}(\Omega)$ is a characteristic function (the general case then follows from the coarea formula). In this case, the left hand side inequality follows from assumption (A), while the other follows from the fact if that $F(u[x+h \Sigma])>0$ for some $x \in I^{h}(B) \cap h \mathbb{Z}^{N}$, then $u$ takes different values on the set $x+h \Sigma$ so that its variation on $B\left(x, \rho_{\Sigma} h\right)$ (where $\rho_{\Sigma}$ is given by (4.2)) is at least $h^{N-1}$.

For every $\nu \in \mathbb{R}^{N}$ we set

The main result of the paper is the following.

$$
F(\nu \cdot \Sigma):=F\left((\nu \cdot y)_{y \in \Sigma}\right) .
$$

Theorem 4.2. Let $\Omega \subseteq \mathbb{R}^{N}$ be a bounded, open and Lipschitz domain, and let $J_{h}:=J_{h}(\cdot, \Omega)$ be defined as in (4.4) for $h>0$. Then the family $\left(J_{h}\right)_{h>0} \Gamma$-converges in the $L^{1}$-topology as $h \rightarrow 0$ to the functional $J: L^{1}(\Omega) \rightarrow[0,+\infty]$ given by

$$
J(u, \Omega)= \begin{cases}\int_{\Omega} F\left(\frac{D u}{|D u|} \cdot \Sigma\right) \mathrm{d}|D u| & \text { if } u \in B V(\Omega) \\ +\infty & \text { if } u \in L^{1}(\Omega) \backslash B V(\Omega),\end{cases}
$$


where for $u \in B V(\Omega)$ the function $D u /|D u|$ stands for the Radon-Nikodym derivative of Du with respect to its total variation $|D u|$.

Since $J(\cdot, \Omega)$ satisfies the generalized coarea formula (see Prop. 3.5), we can also write for $u \in B V(\Omega)$

$$
J(u, \Omega)=\int_{-\infty}^{+\infty} \operatorname{Per}_{\Sigma, F}(\{u>s\}, \Omega) \mathrm{d} s,
$$

where for any finite-perimeter set $E$ in $\Omega$

$$
\operatorname{Per}_{\Sigma, F}(E, \Omega)=\int_{\partial^{*} E \cap \Omega} F\left(\nu_{E} \cdot \Sigma\right) \mathrm{d} \mathcal{H}^{N-1}
$$

In particular

$$
J\left(\chi_{E}, \Omega\right)=\operatorname{Per}_{\Sigma, F}(E, \Omega)
$$

for any finite-perimeter set $E$ in $\Omega$.

The following corollary is a consequence of the $\Gamma$-convergence result of the previous theorem.

Corollary 4.3. Let $\Omega \subseteq \mathbb{R}^{N}$ be a bounded open set with Lipschitz boundary, $g \in L^{\infty}(\Omega)$, and let $u_{h}$ be the solution of

$$
\min _{u \in L^{1}(\Omega)} J_{h}(u, \Omega)+\|u-g\|_{L^{2}(\Omega)}^{2} .
$$

Then $u_{h}$ converges in $L^{1}(\Omega)$ for $h \rightarrow 0$ to the minimizer $u \in B V(\Omega)$ of

$$
\min _{u \in L^{1}(\Omega)} J(u, \Omega)+\|u-g\|_{L^{2}(\Omega)}^{2}
$$

where $J$ is the $\Gamma$-limit of the family $\left(J_{h}\right)_{h>0}$ given by (4.6).

Proof. Without loss of generality we can suppose $u_{h} \in V_{h}(\Omega)$ for any $h>0$. Moreover, as a consequence of the submodularity property (iv) in Proposition 3.2, we have that the functional $J_{h}$ decreases by truncation. This entails that $\left\|u_{h}\right\|_{\infty} \leq\|g\|_{\infty}$ for every $h>0$.

Taking into account (4.5) we get that the total variation of $u_{h}$ is uniformly bounded. By compactness in $B V$, we deduce that there exists $u \in B V(\Omega)$ and a sequence $h_{k} \rightarrow 0$ such that $u_{h_{k}} \rightarrow u$ in $L^{1}(\Omega)$. The convergence is indeed in every $L^{p}$ for every $1 \leq p<+\infty$ since $\left(u_{h}\right)_{h>0}$ is bounded in $L^{\infty}(\Omega)$.

The fact that the limit $u$ is a minimizer of (4.10) is a consequence of Proposition 2.1. Since this minimizer is, in fact, unique, we conclude that the entire family $\left(u_{h}\right)_{h>0}$ converges to $u$ as $h \rightarrow 0$.

Remark 4.4. Notice that equality (4.7) implies that the $\Gamma$-limit $J(u, \Omega)$ satisfies (4.6) for every $u \in B V(\Omega)$. In fact a direct computation shows that (4.6) holds for simple functions. The extension to the whole $B V(\Omega)$ follows by a density argument. Let $u \in B V(\Omega)$, and let $\left(u_{n}\right)_{n \in \mathbb{N}}$ be a sequence of simple functions converging in $L^{1}(\Omega)$ to $u$ and such that $\left|D u_{n}\right|(\Omega) \rightarrow|D u|(\Omega)$ as $n \rightarrow \infty$. From Reshetnyak continuity theorem (see [2], Thm. 2.39) we deduce that

$$
\lim _{n \rightarrow \infty} \int_{\Omega} F\left(\frac{D u_{n}}{\left|D u_{n}\right|} \cdot \Sigma\right) \mathrm{d}\left|D u_{n}\right|=\int_{\Omega} F\left(\frac{D u}{|D u|} \cdot \Sigma\right) \mathrm{d}|D u| .
$$

Since $\left|D u_{n}\right|(\Omega) \rightarrow|D u|(\Omega)$, by coarea formula in $B V(\Omega)$ we get, up to a subsequence, $\operatorname{Per}\left(\left\{u_{n}>s\right\}, \Omega\right) \rightarrow$ $\operatorname{Per}(\{u>s\}, \Omega)$ for a.e. $s \in \mathbb{R}$. Using again Reshetnyak continuity theorem (applied to the measures 
$\nu \mathrm{d} \mathcal{H}^{N-1}\left\llcorner\partial^{*}\left\{u_{n}>s\right\}\right)$, we deduce that

$$
\begin{aligned}
\lim _{n \rightarrow \infty} \operatorname{Per}_{\Sigma, F}\left(\left\{u_{n}>s\right\}, \Omega\right) & =\lim _{n \rightarrow \infty} \int_{\partial^{*}\left\{u_{n}>s\right\} \cap \Omega} F(\nu \cdot \Sigma) \mathrm{d} \mathcal{H}^{N-1} \\
& =\int_{\partial^{*}\{u>s\} \cap \Omega} F(\nu \cdot \Sigma) \mathrm{d} \mathcal{H}^{N-1}=\operatorname{Per}_{\Sigma, F}(\{u>s\}, \Omega) .
\end{aligned}
$$

By the generalized coarea formula for $J$ and the Dominated Convergence Theorem we conclude that

$$
\lim _{n \rightarrow \infty} J\left(u_{n}, \Omega\right)=J(u, \Omega)
$$

so that in view of (4.11), the representation (4.6) is proved.

The rest of the section is devoted to the proof of Theorem 4.2. In view of Proposition 3.5 and of Remark 4.4, in order to study the $\Gamma$-limit of the family $\left(J_{h}\right)_{h>0}$ we can consider the restriction of $J_{h}$ to characteristic functions of sets, and show that it $\Gamma$-converges to the anisotropic perimeter given by (4.8).

By definition, we need to show that given any sequence $h_{m} \downarrow 0$, we have for any Borel set $E \subseteq \Omega$ :

- if $\chi_{E_{m}} \in V_{h_{m}}(\Omega)$ converges to $\chi_{E}$ in $L^{1}(\Omega)$, then

$$
\liminf _{m \rightarrow \infty} J_{h_{m}}\left(\chi_{E_{m}}, \Omega\right) \geq J\left(\chi_{E}, \Omega\right)
$$

- there exists a sequence $\left(E_{m}\right)_{m \in \mathbb{N}}$ with $\chi_{E_{m}} \in V_{h_{m}}(\Omega)$ such that $\chi_{E_{m}} \rightarrow \chi_{E}$ in $L^{1}(\Omega)$ and

$$
\limsup _{m \rightarrow \infty} J_{h_{m}}\left(\chi_{E_{m}}, \Omega\right) \leq J\left(\chi_{E}, \Omega\right) .
$$

We prove inequalities (4.12) and (4.13) in Sections 4.1 and 4.2 respectively. We will use the following "continuous" variant of $J_{h}$, defined on any function and not just on piecewise constant functions of the class $V_{h}$ : we let, for any $u \in L^{1}(\Omega)$,

$$
J_{h}^{c}(u, \Omega):=\frac{1}{h} \int_{I^{h}(\Omega)} F(u[x+h \Sigma]) \mathrm{d} x .
$$

Let $Q_{\nu}$ is the open unit cube centered in 0 with a face orthogonal to $\nu$, and

$$
I_{\nu}:=\left\{x \in \mathbb{R}^{N}: x \cdot \nu>0\right\} .
$$

We have the following result:

Lemma 4.5. There holds

$$
F(\nu \cdot \Sigma)=\lim _{h \rightarrow 0} J_{h}^{c}\left(\chi_{I_{\nu}}, Q_{\nu}\right)
$$

Proof. One has

$$
J_{h}^{c}\left(\chi_{I_{\nu}}, Q_{\nu}\right)=\frac{1}{h} \int_{I^{h}\left(Q_{\nu}\right)} F\left(\chi_{I_{\nu}}[x+h \Sigma]\right) \mathrm{d} x .
$$

Now, letting $u(x):=\nu \cdot x$, we have, for any $s \in \mathbb{R}$ and $x \in \mathbb{R}^{N}, \chi_{I_{\nu}}[x+h \Sigma]=\chi_{\{u>s\}}[x+s \nu+h \Sigma]$, so that we may write

$$
\begin{aligned}
J_{h}^{c}\left(\chi_{I_{\nu}}, Q_{\nu}\right) & =\int_{-1 / 2}^{1 / 2}\left(\frac{1}{h} \int_{I^{h}\left(Q_{\nu}\right)} F\left(\chi_{\{u>s\}}[x+s \nu+h \Sigma]\right) \mathrm{d} x\right) \mathrm{d} s \\
& =\int_{-1 / 2}^{1 / 2}\left(\frac{1}{h} \int_{I^{h}\left(Q_{\nu}\right)+s \nu} F\left(\chi_{\{u>s\}}[y+h \Sigma]\right) \mathrm{d} y\right) \mathrm{d} s .
\end{aligned}
$$


Now, as soon as $|s|<1 / 2-\rho_{\Sigma} h$,

$$
\int_{I^{h}\left(Q_{\nu}\right)+s \nu} F\left(\chi_{\{u>s\}}[y+h \Sigma]\right) \mathrm{d} y=\int_{I^{h}\left(Q_{\nu}\right)} F\left(\chi_{\{u>s\}}[y+h \Sigma]\right) \mathrm{d} y,
$$

and it follows from (4.17) and the coarea formula that

$$
J_{h}^{c}\left(\chi_{I_{\nu}}, Q_{\nu}\right)=J_{h}^{c}\left(u, Q_{\nu}\right)+\epsilon_{h}
$$

where the error $\epsilon_{h} \geq 0$ is

$$
\epsilon_{h}=\frac{1}{h} \int_{\frac{1}{2}-\rho_{\Sigma} h<|s|<\frac{1}{2}}\left(\int_{I^{h}\left(Q_{\nu}\right)+s \nu} F\left(\chi_{\{u>s\}}[y+h \Sigma]\right) \mathrm{d} y-\int_{I^{h}\left(Q_{\nu}\right)} F\left(\chi_{\{u>s\}}[y+h \Sigma]\right) \mathrm{d} y\right) \mathrm{d} s .
$$

One easily checks that $\epsilon_{h} \leq 2\left(\max _{\Sigma} F\right) \rho_{\Sigma}^{2} h \rightarrow 0$ as $h \rightarrow 0$, and (4.16) follows from (4.18) and the observation that

$$
J_{h}^{c}\left(u, Q_{\nu}\right)=F(\nu \cdot \Sigma) \frac{\left|I^{h}\left(Q_{\nu}\right)\right|}{\left|Q_{\nu}\right|} \rightarrow F(\nu \cdot \Sigma)
$$

as $h \rightarrow 0$.

\section{1. $\Gamma$-lim inf inequality}

We prove here the inequality (4.12). First of all, we may assume (upon extracting a subsequence) that the liminf in (4.12) is a limit, and, also, that it is finite (otherwise there is nothing to prove), so that in particular $\sup _{m} J_{h_{m}}\left(\chi_{E_{m}}, \Omega\right)=C<+\infty$. For any $A \subset \subset \Omega$, it follows from (4.5) that for any $m$ large enough, $C_{1}\left|D \chi_{E_{m}}\right|(A) \leq C$ so that (by lower semicontinuity of the total variation) also $E$ must have finite perimeter in $\Omega$.

We consider the non-negative measures

$$
\mu_{m}=h_{m}^{N-1} \sum_{x \in I^{h_{m}}(\Omega) \cap h_{m} \mathbb{Z}^{N}} F\left(\chi_{E_{m}}\left[x+h_{m} \Sigma\right]\right) \delta_{x}
$$

such that $J_{h_{m}}\left(\chi_{E_{m}}, \Omega\right)=\mu_{m}(\Omega)$. Since they are uniformly bounded, we may also assume that there exists a measure $\mu$ such that $\mu_{m} \stackrel{*}{\rightarrow} \mu$ as measures. We therefore have

$$
\mu(\Omega) \leq \liminf _{m \rightarrow \infty} J_{h_{m}}\left(\chi_{E_{m}}, \Omega\right),
$$

hence the thesis follows if we show that $\mu \geq F\left(\nu_{E} \cdot \Sigma\right) \mathcal{H}^{N-1}\left\llcorner\partial^{*} E\right.$ as measures.

It is therefore enough to compute the Radon-Nikodým derivative of the measure $\mu$ with respect to $\mathcal{H}^{N-1}\left\llcorner\partial^{*} E\right.$, and to show it is above $F\left(\nu_{E} \cdot \Sigma\right)$. We know from [2], Thm. 5.52, that it is given, for $\mathcal{H}^{N-1}$-a.e. $x \in \partial^{*} E$, by

$$
\lim _{r \rightarrow 0} \frac{\mu\left(x+r Q_{\nu}\right)}{\mathcal{H}^{N-1}\left(\left(x+r Q_{\nu}\right) \cap \partial^{*} E\right)}
$$

for any $\nu \in \mathbb{R}^{N}$ with unit norm, where $Q_{\nu}$ is as before the open unit cube centered in 0 with a face orthogonal to $\nu$. In particular, at a regular point $x_{0}$ of $\partial^{*} E$ we can choose $\nu=\nu_{E}\left(x_{0}\right)$ (the inner normal to $E$ at $x_{0}$ ) and the limit becomes

$$
\ell=\lim _{r \rightarrow 0} \frac{\mu\left(x_{0}+r Q_{\nu}\right)}{r^{N-1}} .
$$


Let us now show that $\ell \geq F(\nu \cdot \Sigma)$. We can assume $\ell<+\infty$. Notice that since $x_{0}$ is regular, we also have

$$
\lim _{r \rightarrow 0} \frac{1}{r^{N}} \int_{x_{0}+2 r Q_{\nu}}\left|\chi_{I_{\nu}}\left(y-x_{0}\right)-\chi_{E}(y)\right| \mathrm{d} y=0 .
$$

For a.e. $r>0$ (small), we have

$$
\mu\left(x_{0}+r Q_{\nu}\right)=\lim _{m \rightarrow \infty} \mu_{m}\left(x_{0}+r Q_{\nu}\right)
$$

and

$$
\int_{x_{0}+2 r Q_{\nu}}\left|\chi_{I_{\nu}}\left(y-x_{0}\right)-\chi_{E}(y)\right| \mathrm{d} y=\lim _{m \rightarrow \infty} \int_{x_{0}+2 r Q_{\nu}}\left|\chi_{I_{\nu}}\left(y-x_{0}\right)-\chi_{E_{m}}(y)\right| \mathrm{d} y .
$$

Hence, using a diagonal argument, there exist sequences $m_{n}$ and $r_{n}$ such that $h_{m_{n}} / r_{n} \rightarrow 0$,

$$
\ell=\lim _{n \rightarrow \infty} \frac{\mu_{m_{n}}\left(x_{0}+r_{n} Q_{\nu}\right)}{r_{n}^{N-1}}
$$

and

$$
\lim _{n \rightarrow \infty} \frac{1}{r_{n}^{N}} \int_{x_{0}+2 r_{n} Q_{\nu}}\left|\chi_{I_{\nu}}\left(y-x_{0}\right)-\chi_{E_{m_{n}}}(y)\right| \mathrm{d} y=0 .
$$

For each $n$, we now make the change of variable $y=x_{0}+r_{n} z$, and we define $E_{n}^{\prime}=\left(E_{m_{n}}-x_{0}\right) / r_{n} \subset\left(\Omega-x_{0}\right) / r_{n}$. It follows

and

$$
\ell=\lim _{n \rightarrow \infty}\left(\frac{h_{m_{n}}}{r_{n}}\right)^{N-1} \sum_{z \in Q_{\nu} \cap\left[\left(h_{m_{n}} / r_{n}\right) \mathbb{Z}^{N}-x_{0} / r_{n}\right]} F\left(\chi_{E_{n}^{\prime}}\left[z+\left(h_{m_{n}} / r_{n}\right) \Sigma\right]\right)
$$

$$
\lim _{n \rightarrow \infty} \int_{2 Q_{\nu}}\left|\chi_{I_{\nu}}(z)-\chi_{E_{n}^{\prime}}(z)\right| \mathrm{d} z=0 .
$$

We let $h_{n}^{\prime}=h_{m_{n}} / r_{n}$ (which goes to 0 ), and let $\theta_{n}=x_{0} / h_{m_{n}}-\left[x_{0} / h_{m_{n}}\right]$ (the vector whose coordinates are each the fractional part of the corresponding coordinate of $x_{0} / h_{m_{n}}$ ). The limit in (4.21) becomes:

$$
\ell=\lim _{n \rightarrow \infty} h_{n}^{\prime N-1} \sum_{z \in Q_{\nu} \cap h_{n}^{\prime}\left(\mathbb{Z}^{N}-\theta_{n}\right)} F\left(\chi_{E_{n}^{\prime}}\left[z+h_{n}^{\prime} \Sigma\right]\right) .
$$

Letting $E_{n}^{\prime \prime}:=E_{n}^{\prime}+h_{n}^{\prime} \theta_{n}$, we clearly still have

$$
\lim _{n \rightarrow \infty} \int_{Q_{\nu}}\left|\chi_{I_{\nu}}(z)-\chi_{E_{n}^{\prime \prime}}(z)\right| \mathrm{d} z=0 .
$$

From now on, to simplify, we will denote $E_{n}^{\prime \prime}$ and $h_{n}^{\prime}$ by, respectively, $E_{n}$ and $h_{n}$. We consider a basis $\left(\nu_{1}, \ldots, \nu_{N}\right)$ of $\mathbb{R}^{N}$ such that each $\nu_{i}$ is orthogonal to a face of $Q_{\nu}$, and with $\nu_{N}=\nu$. We choose $\eta_{0}>0$ (small). Writing $x=s \nu_{1}+x^{\prime}=\left(s, x^{\prime}\right)$ with $x^{\prime} \cdot \nu_{1}=0$, we have (using Fubini's Theorem)

$$
\begin{aligned}
\int_{0}^{\eta_{0}}\left[\frac{1}{h_{n}} \int_{1 / 2-\eta-\rho_{\Sigma} h_{n}}^{1 / 2-\eta} \int_{[-1 / 2,1 / 2]^{N-1}} \mid \chi_{I_{\nu}}(x)\right. & \left.-\chi_{E_{n}}(x) \mid \mathrm{d} x^{\prime} \mathrm{d} s\right] \mathrm{d} \eta \\
& =\rho_{\Sigma} \int_{1 / 2-\eta_{0}-\rho_{\Sigma} h_{n}}^{1 / 2} \int_{[-1 / 2,1 / 2]^{N-1}}\left|\chi_{I_{\nu}}(x)-\chi_{E_{n}}(x)\right| \mathrm{d} x^{\prime} \mathrm{d} s
\end{aligned}
$$

which, thanks to (4.24), goes to 0 . 
Hence, up to a subsequence, we may assume that for a.e. $\eta \in\left[0, \eta_{0}\right]$,

$$
\frac{1}{h_{n}} \int_{1 / 2-\eta-\rho_{\Sigma} h_{n}}^{1 / 2-\eta} \int_{Q_{\nu} \cap \nu_{1}^{\perp}}\left|\chi_{I_{\nu}}\left(s \nu_{1}+x^{\prime}\right)-\chi_{E_{n}}\left(s \nu_{1}+x^{\prime}\right)\right| \mathrm{d} x^{\prime} \mathrm{d} s \rightarrow 0
$$

as $n \rightarrow \infty$. The same holds if we replace in (4.26) $\nu_{1}$ with any of the $\nu_{i}, i=2, \ldots, N-1$, or if we replace the interval $\left[1 / 2-\eta-\rho_{\Sigma} h_{n}, 1 / 2-\eta\right]$ of integration in the first integral with the interval $\left[-1 / 2+\eta,-1 / 2+\eta+\rho_{\Sigma} h_{n}\right]$. Let us therefore choose a $\eta>0$ such that all the above mentioned limits are 0 , and let $Q_{\nu}^{\eta}=(1-2 \eta) Q_{\nu}$.

We now extend periodically $E_{n} \cap Q_{\nu}^{\eta}$ in the directions $\nu_{1}, \ldots, \nu_{N-1}$, into a set $\hat{E}_{n} \subset \nu^{\perp}+[-1 / 2+\eta, 1 / 2-\eta] \nu$, by letting $\hat{x} \in \hat{E}_{n}$ if and only if $\hat{x}=x+(1-2 \eta) \sum_{i=1}^{N-1} k_{i} \nu_{i}$ for some point $x \in E_{n} \cap Q_{\nu}^{\eta}$ and integers $k_{i} \in \mathbb{Z}$. It follows from (4.23) that

$$
\ell \geq \liminf _{n \rightarrow \infty} J_{h_{n}}^{c}\left(\chi_{\hat{E}_{n}}, Q_{\nu}^{\eta}\right)
$$

where $J_{h_{n}}^{c}$ is as in (4.14), and (recalling (4.5)),

$$
\sup _{n \in \mathbb{N}}\left|D \chi_{\hat{E}_{n}}\right|\left(\overline{Q_{\nu}^{\eta}}\right) \leq \sup _{n \in \mathbb{N}}\left|D \chi_{E_{n}}\right|\left(\operatorname{int}\left(Q_{\nu}^{\eta}\right)\right)+2 N<+\infty .
$$

We claim that for any $\tau \in \nu^{\perp}$, we also have

$$
J_{h_{n}}^{c}\left(\chi_{\hat{E}_{n}}, Q_{\nu}^{\eta}\right) \geq J_{h_{n}}^{c}\left(\chi_{\hat{E}_{n}}, \tau+Q_{\nu}^{\eta}\right)-\epsilon_{n}
$$

for some error $\epsilon_{n} \rightarrow 0$ which is independent on $\tau$.

Assume to simplify that $\tau=s \nu_{1}$ for some $s \in \mathbb{R}$. If $s$ is $(1-2 \eta)$ times an integer, then (4.29) is obvious. If not, we may assume without loss of generality that $0<s<1-2 \eta$. We have

$$
J_{h_{n}}^{c}\left(\chi_{\hat{E}_{n}}, s \nu_{1}+Q_{\nu}^{\eta}\right)=\frac{1}{h_{n}} \int_{I^{h_{n}\left(s \nu_{1}+Q_{\nu}^{\eta}\right)}} F\left(\chi_{\hat{E}_{n}}\left[x+h_{n} \Sigma\right]\right) \mathrm{d} x .
$$

The domain of integration is split into three parts $D_{1}=I^{h_{n}}\left(s \nu_{1}+Q_{\nu}^{\eta}\right) \cap I^{h_{n}}\left(Q_{\nu}^{\eta}\right), D_{2}=I^{h_{n}}\left(s \nu_{1}+Q_{\nu}^{\eta}\right) \cap I^{h_{n}}((1-$ $\left.2 \eta) \nu_{1}+Q_{\nu}^{\eta}\right)$, and $D_{3}=I^{h_{n}}\left(s \nu_{1}+Q_{\nu}^{\eta}\right) \backslash\left(D_{1} \cup D_{2}\right)$. Since $D_{1}$ and $\left(D_{2}-(1-2 \eta) \nu_{1}\right)$ are disjoint subsets of $I^{h_{n}}\left(Q_{\nu}^{\eta}\right)$, it follows

$$
J_{h_{n}}^{c}\left(\chi_{\hat{E}_{n}}, s \nu_{1}+Q_{\nu}^{\eta}\right) \leq J_{h_{n}}^{c}\left(\chi_{\hat{E}_{n}}, Q_{\nu}^{\eta}\right)+\frac{1}{h_{n}} \int_{D_{3}} F\left(\chi_{\hat{E}_{n}}\left[x+h_{n} \Sigma\right]\right) \mathrm{d} x .
$$

We have $D_{3} \subset S_{1}^{+} \cup\left((1-2 \eta) \nu_{1}+S_{1}^{-}\right)$, where for $i=1, \ldots, N-1$,

$$
S_{i}^{ \pm}=\left\{x=\sum_{j=1}^{N} x_{j} \nu_{j}: \frac{1}{2}-\eta-\rho_{\Sigma} h_{n} \leq \pm x_{i}<\frac{1}{2}-\eta,\left|x_{j}\right|<\frac{1}{2}-\eta \forall j \neq i\right\} .
$$

Let us show that $\left(1 / h_{n}\right) \int_{S_{1}^{+}} F\left(\chi_{\hat{E}_{n}}\left[x+h_{n} \Sigma\right]\right) \mathrm{d} x \rightarrow 0$ as $n \rightarrow \infty$. We have, using the notation $x=s \nu_{1}+x^{\prime}$, $x^{\prime} \cdot \nu_{1}=0$, and the change of variable $s=1 / 2-\eta-h_{n} \xi$,

$$
\frac{1}{h_{n}} \int_{S_{1}^{+}} F\left(\chi_{\hat{E}_{n}}\left[x+h_{n} \Sigma\right]\right) \mathrm{d} x=\int_{0}^{\rho_{\Sigma}} \int_{[-1 / 2+\eta, 1 / 2-\eta]^{N-1}} F\left(v_{n}\left(\xi, x^{\prime}\right)\right) \mathrm{d} x^{\prime} \mathrm{d} \xi
$$

where for each $\xi, x^{\prime}, v_{n}\left(\xi, x^{\prime}\right) \in\{0,1\}^{\Sigma}$ is the vector $\chi_{\hat{E}_{n}}\left[\left(1 / 2-\eta-h_{n} \xi\right) \nu_{1}+x^{\prime}+h_{n} \Sigma\right]$. We observe that from (4.26), we have (using the same change of variable, and observing that $\chi_{I_{\nu}}$ depends only on $x_{N}^{\prime}$ )

$$
\int_{0}^{\rho_{\Sigma}} \int_{[-1 / 2,1 / 2]^{N-1}}\left|\chi_{I_{\nu}}\left(x_{N}^{\prime}\right)-\chi_{E_{n}}\left(1 / 2-\eta-h_{n} \xi, x^{\prime}\right)\right| \mathrm{d} x^{\prime} \mathrm{d} \xi \rightarrow 0
$$


as $n \rightarrow \infty$. In particular, up to a subsequence, we may assume that each component of the vector $v_{n}\left(\xi, x^{\prime}\right)$ converges to $\chi_{I_{\nu}}\left(x_{N}^{\prime}\right)$ as $n \rightarrow \infty$, for a.e. $\left(\xi, x^{\prime}\right)$. Notice that these components take only the value 0 or 1 , hence, they must be equal to $\chi_{I_{\nu}}\left(x_{N}^{\prime}\right)$ for $n$ large enough. Since $F(0)=F\left(\chi_{\Sigma}\right)=0$, it follows that $F\left(v_{n}\left(\xi, x^{\prime}\right)\right) \rightarrow 0$ a.e., and since $F$ is bounded we find (using Lebesgue's theorem) that the integral in the right-hand side of (4.31) goes to 0 .

In the same way, we can show that $\left(1 / h_{n}\right) \int_{S_{1}^{-}} F\left(\chi_{\hat{E}_{n}}\left[x+h_{n} \Sigma\right]\right) \mathrm{d} x \rightarrow 0$, so that (4.29) follows from (4.30) and the inclusion $D_{3} \subset S_{1}^{+} \cup\left((1-2 \eta) \nu_{1}+S_{1}^{-}\right)$. In the general case (if $\tau$ is not parallel to $\nu_{1}$ ), we can show in the same way that (4.29) holds provided

$$
\epsilon_{n}=\frac{1}{h_{n}} \sum_{i=1}^{N-1} \int_{S_{i}^{-} \cup S_{i}^{+}} F\left(\chi_{\hat{E}_{n}}\left[x+h_{n} \Sigma\right]\right) \mathrm{d} x ;
$$

our choice of $\eta$ guarantees that $\epsilon_{n}$ still goes to zero.

Let $u_{n}: Q_{\nu}^{\eta} \rightarrow[0,1]$ be the average of $\chi_{\hat{E}_{n}}$ on each hyperplane orthogonal to $\nu$, given by

$$
u_{n}(x):=\frac{1}{(1-2 \eta)^{N-1}} \int_{Q_{\nu}^{\eta} \cap \nu^{\perp}} \chi_{\hat{E}_{n}}\left(x+x^{\prime}\right) \mathrm{d} x^{\prime} .
$$

It is clear that $u_{n}$ depends only on $x \cdot \nu$. Since

$$
\int_{Q_{\nu}^{\eta}}\left|u_{n}-\chi_{I_{\nu}}\right| \mathrm{d} x \leq \int_{Q_{\nu}^{\eta}}\left|\chi_{\hat{E}_{n}}-\chi_{I_{\nu}}\right| \mathrm{d} x
$$

we deduce

$$
u_{n} \rightarrow \chi_{I_{\nu}} \quad \text { in } L^{1}\left(Q_{\nu}^{\eta}\right)
$$

Notice that for a.e. $x \in Q_{\nu}^{\eta}$, the vector $u_{n}\left[x+h_{n} \Sigma\right]$ is also the average on the hyperplane through $x$ orthogonal to $\nu$ of the vectors $\chi_{\hat{E}_{n}}\left[\cdot+h_{n} \Sigma\right]$, so that, by Jensen's inequality and using (4.29)

$$
J_{h_{n}}^{c}\left(\chi_{\hat{E}_{n}}, Q_{\nu}^{\eta}\right) \geq J_{h_{n}}^{c}\left(u_{n}, Q_{\nu}^{\eta}\right)-\epsilon_{n} .
$$

Together with (4.27), it yields

$$
\ell \geq \liminf _{n \rightarrow \infty} J_{h_{n}}^{c}\left(u_{n}, Q_{\nu}^{\eta}\right)
$$

It is clear that (4.28) also yields a uniform bound on the total variations $\left|D u_{n}\right|\left(Q_{\nu}^{\eta}\right), n \in \mathbb{N}$. Let us fix $\varepsilon, \delta \in] 0,1\left[\right.$. By the generalized coarea formula for $J_{h_{n}}^{c}$, and the coarea formula in $B V$ we get

$$
\begin{aligned}
C \geq J_{h_{n}}^{c}\left(u_{n}, Q_{\nu}^{\eta}\right)+\varepsilon\left|D u_{n}\right|\left(Q_{\nu}^{\eta}\right) & =\int_{0}^{1}\left[J_{h_{n}}^{c}\left(\chi_{\left\{u_{n}>s\right\}}, Q_{\nu}^{\eta}\right)+\varepsilon \mathcal{H}^{N-1}\left(\partial^{*}\left\{u_{n}>s\right\} \cap Q_{\nu}^{\eta}\right)\right] \mathrm{d} s \\
& \geq \int_{\delta}^{1-\delta}\left[J_{h_{n}}^{c}\left(\chi_{\left\{u_{n}>s\right\}}, Q_{\nu}^{\eta}\right)+\varepsilon \mathcal{H}^{N-1}\left(\partial^{*}\left\{u_{n}>s\right\} \cap Q_{\nu}^{\eta}\right)\right] \mathrm{d} s
\end{aligned}
$$

for some positive constant $C$. We deduce that there exists $\left.s_{n} \in\right] \delta, 1-\delta[$ such that

$$
J_{h_{n}}^{c}\left(u_{n}, Q_{\nu}^{\eta}\right)+\varepsilon\left|D u_{n}\right|\left(Q_{\nu}^{\eta}\right) \geq(1-2 \delta) J_{h_{n}}^{c}\left(\chi_{\left\{u_{n}>s_{n}\right\}}, Q_{\nu}^{\eta}\right)
$$

and

We have

$$
C \geq \varepsilon(1-2 \delta) \mathcal{H}^{N-1}\left(\partial^{*}\left\{u_{n}>s_{n}\right\} \cap Q_{\nu}^{\eta}\right)
$$

$$
v_{n}:=\chi_{\left\{u_{n}>s_{n}\right\}} \rightarrow \chi_{I_{\nu}} \quad \text { in } L^{1}\left(Q_{\nu}^{\eta}\right) .
$$


By (4.34) and since $u_{n}$ depends only on $x \cdot \nu$, we deduce that there exists, up to a subsequence, an odd number $M$ independent of $n$ such that

with

$$
\partial^{*}\left\{v_{n}=1\right\}=Q_{\nu}^{\eta} \cap \bigcup_{k=1}^{M}\left(a_{n}^{k} \nu+\nu^{\perp}\right)
$$

$$
-\frac{1}{2}+\eta<a_{n}^{1}<a_{n}^{2}<\ldots<a_{n}^{M}<\frac{1}{2}-\eta .
$$

Without loss of generality, we can assume that $a_{n}^{k} \rightarrow 0$ for every $k=1, \ldots, M, v_{n}=0$ if $x \cdot \nu<a_{n}^{1}$ and $v_{n}=1$ if $x \cdot \nu>a_{n}^{M}$. Indeed, if some of the points $a_{n}^{k}$ do not go to zero as $n \rightarrow \infty$, we can lower the energy $J_{h_{n}}^{c}\left(v_{n}, Q_{\nu}^{\eta}\right)$ by "removing" from $v_{n}$ the corresponding discontinuities.

If $M>1$, let us consider the function $\tilde{v}_{n}=\chi_{\left\{x \cdot \nu>a_{n}^{2}\right\}}$. Since $v_{n} \vee \tilde{v}_{n}$ is both a translate of $\tilde{v}_{n}$ (of $\left(a_{n}^{1}-a_{n}^{2}\right) \nu$ ) and of $\chi_{I_{\nu}}$ (of $a_{n}^{2} \nu$ ), for $n$ large we have

$$
J_{h_{n}}^{c}\left(\tilde{v}_{n}, Q_{\nu}^{\eta}\right)=J_{h_{n}}^{c}\left(v_{n} \vee \tilde{v}_{n}, Q_{\nu}^{\eta}\right)=J_{h_{n}}^{c}\left(\chi_{I_{\nu}}, Q_{\nu}^{\eta}\right) .
$$

By the submodularity property of $F$, we obtain setting $v_{n}^{1}:=v_{n} \wedge \tilde{v}_{n}$ that for $n$ large enough,

$$
J_{h_{n}}^{c}\left(v_{n}^{1}, Q_{\nu}^{\eta}\right) \leq J_{h_{n}}^{c}\left(v_{n}, Q_{\nu}^{\eta}\right)
$$

If $M=3$ then $v_{n}^{1}=\chi_{\left\{x \cdot \nu>a_{n}^{3}\right\}}$ is a translate of $\chi_{I_{\nu}}$. If $M>3$, then we can reiterate this argument, replacing now $a_{n}^{2}$ with $a_{n}^{4}$ : after a finite number of steps we eventually find a translate of $\chi_{I_{\nu}}$ with energy lower than $J_{h_{n}}^{c}\left(v_{n}, Q_{\nu}^{\eta}\right)$. Hence, for $n$ large enough,

$$
J_{h_{n}}^{c}\left(\chi_{I_{\nu}}, Q_{\nu}^{\eta}\right) \leq J_{h_{n}}^{c}\left(v_{n}, Q_{\nu}^{\eta}\right) .
$$

By (4.16) and a straightforward scaling argument, the left-hand side of $(4.35)$ goes to $(1-2 \eta)^{N-1} F(\nu \cdot \Sigma)$ as $n \rightarrow \infty$. Recalling (4.32) and (4.33), we deduce

$$
\ell+\varepsilon \sup _{n \in \mathbb{N}}\left|D u_{n}\right|\left(Q_{\nu}^{\eta}\right) \geq(1-2 \delta)(1-2 \eta)^{N-1} F(\nu \cdot \Sigma),
$$

and since $\varepsilon, \delta$ and $\eta$ can be chosen arbitrarily small we deduce that $\ell \geq F(\nu \cdot \Sigma)$. It follows that $\mu \geq$ $F(\nu \cdot \Sigma) \mathcal{H}^{N-1}\left\llcorner\partial^{*} E\right.$, which together with (4.19) yields (4.12).

\section{2. $\Gamma$-lim sup inequality}

Let us now show the inequality (4.13). We first show the following generalization of the formula (4.16):

Lemma 4.6. Let $A \subset \Omega$ be an open set, and assume $\mathcal{H}^{N-1}\left(\partial I_{\nu} \cap \partial A\right)=0$. Then

$$
\lim _{h \rightarrow 0} J_{h}^{c}\left(\chi_{I_{\nu}}, A\right)=\mathcal{H}^{N-1}\left(\partial I_{\nu} \cap A\right) F(\nu \cdot \Sigma)
$$

Proof. Let $\varepsilon>0$. Let $\left(\nu_{i}\right)_{i=1}^{N}$ be a basis of $\mathbb{R}^{N}$, with $\nu_{N}=\nu$, and let us consider the family $\mathcal{Q}_{\varepsilon}$ of all cubes $Q \subset A$, of side $\varepsilon$, centered at the points $\sum_{i=1}^{N} \varepsilon k_{i} \nu_{i}$ for $k_{i} \in \mathbb{Z}^{N}$, and such that each face is orthogonal to some $\nu_{i}$.

We have obviously

$$
\lim _{h \rightarrow 0} J_{h}^{c}\left(\chi_{I_{\nu}}, A\right) \geq \sum_{Q \in \mathcal{Q}_{\varepsilon}} \liminf _{h \rightarrow 0} J_{h}^{c}\left(\chi_{I_{\nu}}, Q\right)
$$

while (by (4.16) and a simple scaling argument) for each $Q$,

$$
\lim _{h \rightarrow 0} J_{h}^{c}\left(\chi_{I_{\nu}}, Q\right)=\varepsilon^{N-1} F(\nu \cdot \Sigma) .
$$


Hence,

$$
\lim _{h \rightarrow 0} J_{h}^{c}\left(\chi_{I_{\nu}}, A\right) \geq \mathcal{H}^{N-1}\left(\partial I_{\nu} \cap \bigcup_{Q \in \mathcal{Q}_{\varepsilon}} Q\right) F(\nu \cdot \Sigma)
$$

letting then $\varepsilon \rightarrow 0$, it shows " $\geq$ " in (4.36).

To show the reverse inequality, we let, for each $\varepsilon>0, A^{\varepsilon}=\bigcup_{Q \in \mathcal{Q}_{\varepsilon}} Q$, and first observe that for any $\eta>0$,

$$
J_{h}^{c}\left(\chi_{I_{\nu}}, A^{\varepsilon}\right) \leq \sum_{Q \in \mathcal{Q}_{\varepsilon}} J_{h}^{c}\left(\chi_{I_{\nu}},(1+\eta) Q\right)
$$

as soon as $h<\eta \varepsilon /\left(2 \rho_{\Sigma}\right)$, where here $(1+\eta) Q$ denotes the cube of same center as $Q$, and dilated by the factor $1+\eta$. Taking the limit $h \rightarrow 0$, we find

$$
\limsup _{h \rightarrow 0} J_{h}^{c}\left(\chi_{I_{\nu}}, A^{\varepsilon}\right) \leq(1+\eta)^{N-1} \mathcal{H}^{N-1}\left(\partial I_{\nu} \cap A^{\varepsilon}\right) F(\nu \cdot \Sigma) \leq \mathcal{H}^{N-1}\left(\partial I_{\nu} \cap A\right) F(\nu \cdot \Sigma)+C \eta
$$

for any $\eta>0$. Now,

$$
J_{h}^{c}\left(\chi_{I_{\nu}}, A\right)=J_{h}^{c}\left(\chi_{I_{\nu}}, A^{\varepsilon}\right)+\frac{1}{h} \int_{I^{h}(A) \backslash I^{h}\left(A^{\varepsilon}\right)} F\left(\chi_{I_{\nu}}[x+h \Sigma]\right) \mathrm{d} x
$$

so that (4.36) will follow from (4.37) if we show that

$$
\frac{1}{h} \int_{I^{h}(A) \backslash I^{h}\left(A^{\varepsilon}\right)} F\left(\chi_{I_{\nu}}[x+h \Sigma]\right) \mathrm{d} x \rightarrow 0
$$

as $h \rightarrow 0$ and $\varepsilon \rightarrow 0$. The integrand above is zero when $x$ is at distance larger than $h \rho_{\Sigma}$ to the interface $\partial I_{\nu}$, and $x \in A$ is out of the domain of integration as soon as it is at distance larger than $h \rho_{\Sigma}$ to $A \backslash A^{\varepsilon}$, for instance when $x \in A_{2 \varepsilon}:=\{\xi \in A: \operatorname{dist}(\xi, \partial A)>2 \varepsilon\}$. Hence, the error (4.38) is bounded by

$$
\left(\max _{\Sigma} F\right) \frac{\left|\left(A \backslash A_{2 \varepsilon}\right) \cap\left(\partial I_{\nu}+\left[-h \rho_{\Sigma}, h \rho_{\Sigma}\right] \nu\right)\right|}{h} .
$$

Let $G \subset \partial I_{\nu}$ be a relatively open set which contains $\partial I_{\nu} \cap \partial A$. We claim that there exists $\delta_{0}>0$ small such that the projection of $\left(A \backslash A_{\delta}\right) \cap\left(\partial I_{\nu}+[-\delta, \delta] \nu\right)$ onto $\partial I_{\nu}$ is contained in $G$ for any $\delta<\delta_{0}$ : if not, one finds a sequence $\delta_{n} \rightarrow 0$ and points $x_{n} \in\left(A \backslash A_{\delta_{n}}\right) \cap\left(\partial I_{\nu}+\left[-\delta_{n}, \delta_{n}\right] \nu\right)$ which project outside of $G$, but then, any limit point of this sequence should be in $\partial A \cap \partial I_{\nu}$ but outside $G$, which is not possible.

Then, if we choose $\varepsilon<\delta_{0} / 2 \sqrt{N}$, and $h$ small enough, we have

$$
\left(A \backslash A_{2 \varepsilon}\right) \cap\left(\partial I_{\nu}+\left[-h \rho_{\Sigma}, h \rho_{\Sigma}\right] \nu\right) \subset G+\left[-h \rho_{\Sigma}, h \rho_{\Sigma}\right] \nu
$$

so that the limsup of (4.39), as $h \rightarrow 0$, is less than

$$
\rho_{\Sigma}\left(\max _{\Sigma} F\right) \mathcal{H}^{N-1}(G)
$$

Since we assumed that $\mathcal{H}^{N-1}\left(\partial I_{\nu} \cap \partial A\right)=0, \mathcal{H}^{N-1}(G)$ may be chosen as small as we wish so that (4.38) holds. Hence the lemma is proved. 
Now, we show the following estimate:

Lemma 4.7. Let $A \subset \Omega$ be an open set with continuous boundary, and let $E \subset \Omega$ be a finite-perimeter set such that $\mathcal{H}^{N-1}\left(\overline{\partial^{*} E \cap A} \backslash \partial^{*} E \cap A\right)=0$. Then,

$$
\limsup _{h \rightarrow 0} J_{h}^{c}\left(\chi_{E}, A\right) \leq\left(2 \rho_{\Sigma} \max _{\Sigma} F\right) \mathcal{H}^{N-1}\left(\partial^{*} E \cap A\right)
$$

Proof. Since $A$ has a continuous boundary, for $h$ small enough, $x+h \Sigma$ contains points of Lebesgue density of $E$ both zero and one only if $x$ is close enough to $\partial^{*} E \cap A$, namely, dist $\left(x, \partial^{*} E \cap A\right) \leq h \rho_{\Sigma}$. Hence,

$$
J_{h}^{c}\left(\chi_{E}, A\right)=\frac{1}{h} \int_{I^{h}(A)} F\left(\chi_{E}[x+h \Sigma]\right) \mathrm{d} x \leq \frac{\left|\left\{x \in A: \operatorname{dist}\left(x, \partial^{*} E \cap A\right) \leq h \rho_{\Sigma}\right\}\right|}{h}\left(\max _{\Sigma} F\right) .
$$

By standard results on the Minkowski contents [9], Theorem 3.2.39, the last fraction goes to $2 \rho_{\Sigma} \mathcal{H}^{N-1}\left(\partial^{*} E \cap A\right)$ as $h \rightarrow 0$, which shows the lemma.

We deduce the following:

Corollary 4.8. Let $\Omega \subset \mathbb{R}^{N}$ have a continuous boundary, and let $E \subset \Omega$ be a set whose boundary is made of a finite union of subsets of $(N-1)$-dimensional hyperplanes. Then

$$
\lim _{h \rightarrow 0} J_{h}^{c}\left(\chi_{E}, \Omega\right)=\int_{\partial E \cap \Omega} F\left(\nu_{E}(x) \cdot \Sigma\right) \mathrm{d} \mathcal{H}^{N-1}(x)
$$

where $\nu_{E}(x)$ is the inner normal to $E$ at $x$.

Proof. By assumption, $\partial E \cap \Omega=\bigcup_{i=1}^{M} P_{i}$ where $P_{i} \subset\left(x_{i}+\partial I_{\nu_{i}}\right)$ for some $x_{i} \in \mathbb{R}^{N}$ and $\nu_{i} \in \mathbb{R}^{N}$ with unit norm, moreover we assume that $\nu_{i}=\nu_{E}$ on $P_{i}\left(\nu_{i}\right.$ points towards the interior of $E$ ). (We also assume that the $P_{i}$ are "maximal", in the sense that $\bar{P}_{i} \cap \bar{P}_{j} \neq \emptyset \Rightarrow \nu_{i} \neq \nu_{j}$ for any $i \neq j$.) Let $S=\Omega \cap \bigcup_{i=1}^{M} \partial P_{i}$, where the $\partial P_{i}$ is the relative boundary of the face inside the hyperplane $\left(x_{i}+\partial I_{\nu_{i}}\right)$ : it is a $(N-2)$-dimensional set, with $\mathcal{H}^{N-2}(S)<+\infty$.

We choose $\varepsilon>0$ and build a finite covering $\left(A_{i}\right)_{i=1}^{M+2}$ of $\partial E \cap \Omega$ with bounded open sets with continuous boundary, as follows: $A_{M+1}=\{x \in \Omega$ : $\operatorname{dist}(x, \partial \Omega)<\varepsilon\}, A_{M+2}=\{x \in \Omega$, : $\operatorname{dist}(x, S)<\varepsilon\}$, and $A_{i}$ is a neighborhood of $P_{i} \backslash\left(A_{M+1} \cup A_{M+2}\right)$ which does not intersect $P_{j}$ for $j \neq i$. By Lemmas 4.6 and 4.7 ,

$$
\left|\lim _{h \rightarrow 0} J_{h}^{c}\left(\chi_{E}, \Omega\right)-\sum_{i=1}^{M} \mathcal{H}^{N-1}\left(P_{i} \cap A_{i}\right) F\left(\nu_{i} \cdot \Sigma\right)\right| \leq C\left(\mathcal{H}^{N-1}\left(\partial E \cap A_{M+1}\right)+\mathcal{H}^{N-1}\left(\partial E \cap A_{M+2}\right)\right)
$$

with $C=2 \rho_{\Sigma} \max _{\Sigma} F$. Letting $\varepsilon \rightarrow 0$ shows the corollary.

We are now able to show the following proposition, which essentially shows the $\Gamma$-convergence of $J_{h}$ to $J$ on the restricted class of polyhedral sets.

Proposition 4.9. Let $\Omega \subset \mathbb{R}^{N}$ have a continuous boundary, and let $E \subset \Omega$ be a set whose boundary is made of a finite union of subsets of $(N-1)$-dimensional hyperplanes. Then, there exist sets $E_{h}$ with $\chi_{E_{h}} \in V_{h}(\Omega)$, $\chi_{E_{h}} \rightarrow \chi_{E}$ in $L^{1}(\Omega)$ as $h \rightarrow 0$, and

$$
\lim _{h \rightarrow 0} J_{h}\left(\chi_{E_{h}}, \Omega\right)=\int_{\partial E \cap \Omega} F\left(\nu_{E}(x) \cdot \Sigma\right) \mathrm{d} \mathcal{H}^{N-1}(x)=J\left(\chi_{E}, \Omega\right) .
$$


Proof. We have, making in (4.14) the change of variable $x=z+y$ with $z \in h \mathbb{Z}^{N}$ and $y \in\left[0, h\left[{ }^{N}\right.\right.$,

$$
J_{h}^{c}\left(\chi_{E}, \Omega\right)=\frac{1}{h^{N}} \int_{\left[0, h\left[^{N}\right.\right.}\left[h^{N-1} \sum_{z \in\left(I^{h}(\Omega)-y\right) \cap h \mathbb{Z}^{N}} F\left(\chi_{E}[y+z+h \Sigma]\right)\right] \mathrm{d} y
$$

so that (by Cor. 4.8) we can choose for each $h$ a $y_{h} \in\left[0, h\left[^{N}\right.\right.$ such that

$$
\limsup _{h \rightarrow 0} h^{N-1} \sum_{z \in\left(I^{h}(\Omega)-y_{h}\right) \cap h \mathbb{Z}^{N}} F\left(\chi_{E}\left[y_{h}+z+h \Sigma\right]\right) \leq J\left(\chi_{E}, \Omega\right),
$$

we can assume moreover that no point in $y_{h}+h \mathbb{Z}^{N} \cap \Omega$ lies on $\partial E$.

For each $\varepsilon>0$ we let $\Omega_{\varepsilon}=\{x \in \Omega$ : dist $(x, \partial \Omega)>\varepsilon\}$. We fix $\varepsilon>0$, and define $E_{h}^{\varepsilon}$ as follows (for $h$ small enough):

$$
\chi_{E_{h}^{\varepsilon}}=\sum_{z \in \Omega_{\varepsilon} \cap h \mathbb{Z}^{N}} \chi_{E}\left(y_{h}+z\right) \chi_{Q_{z}^{h}}+\sum_{z \in\left(\Omega \backslash \Omega_{\varepsilon}\right) \cap h \mathbb{Z}^{N}} \chi_{E}(z) \chi_{Q_{z}^{h}}
$$

where $Q_{z}^{h}$ is the cube defined in (4.3). It is not difficult to show that $\chi_{E_{h}^{\varepsilon}} \rightarrow \chi_{E}$ in $L^{1}$ as $h \rightarrow 0$, in fact, it converges locally uniformly in $\Omega \backslash \partial E$. We have if $h$ is small enough

$$
\begin{aligned}
J_{h}\left(\chi_{E_{h}^{\varepsilon}}, \Omega\right) \leq & h^{N-1} \sum_{x \in I^{h}\left(\Omega_{\varepsilon}\right) \cap h \mathbb{Z}^{N}} F\left(\chi_{E_{h}^{\varepsilon}}[x+h \Sigma]\right)+h^{N-1} \sum_{x \in I^{h}\left(\Omega \backslash \Omega_{2 \varepsilon}\right) \cap h \mathbb{Z}^{N}} F\left(\chi_{E_{h}^{\varepsilon}}[x+h \Sigma]\right) \\
& \leq h^{N-1} \sum_{z \in\left(I^{h}(\Omega)-y_{h}\right) \cap h \mathbb{Z}^{N}} F\left(\chi_{E}\left[y_{h}+z+h \Sigma\right]\right)+h^{N-1} \sum_{x \in I^{h}\left(\Omega \backslash \Omega_{2 \varepsilon}\right) \cap h \mathbb{Z}^{N}} F\left(\chi_{E_{h}^{\varepsilon}}[x+h \Sigma]\right) .
\end{aligned}
$$

Now, thanks to (4.41) we get

$$
\limsup _{h \rightarrow 0} J_{h}\left(\chi_{E_{h}^{\varepsilon}}, \Omega\right) \leq J\left(\chi_{E}, \Omega\right)+\limsup _{h \rightarrow 0} h^{N-1} \sum_{x \in I^{h}\left(\Omega \backslash \Omega_{2 \varepsilon}\right) \cap h \mathbb{Z}^{N}} F\left(\chi_{E_{h}^{\varepsilon}}[x+h \Sigma]\right) .
$$

In the sum, on the other hand, $F\left(\chi_{E_{h}^{\varepsilon}}[x+h \Sigma]\right)$ is not zero only when some point of $x+h \Sigma$ lies in $E_{h}^{\varepsilon}$ and some other in $\Omega \backslash E_{h}^{\varepsilon}$, and such $x$ are at distance at most $h\left(1+\rho_{\Sigma}\right)$ from $\partial E \cap \Omega$, so that

$$
Q_{x}^{h} \subset\left\{\xi \in \Omega \backslash \Omega_{2 \varepsilon+h \sqrt{N}}: \operatorname{dist}(\xi, \partial E \cap \Omega) \leq h\left(1+\sqrt{N}+\rho_{\Sigma}\right)\right\} .
$$

Therefore, the sum is bounded by

$$
\frac{\left|\left\{\xi \in \Omega \backslash \Omega_{2 \varepsilon+h \sqrt{N}}: \operatorname{dist}(\xi, \partial E \cap \Omega) \leq h\left(1+\sqrt{N}+\rho_{\Sigma}\right)\right\}\right|}{h},
$$

which goes to $2\left(1+\sqrt{N}+\rho_{\Sigma}\right) \mathcal{H}^{N-1}\left(\partial E \cap\left(\Omega \backslash \Omega_{2 \varepsilon}\right)\right)$ as $h \rightarrow 0$. We deduce from (4.43) that

$$
\limsup _{h \rightarrow 0} J_{h}\left(\chi_{E_{h}^{\varepsilon}}, \Omega\right) \leq J\left(\chi_{E}, \Omega\right)+C \mathcal{H}^{N-1}\left(\partial E \cap\left(\Omega \backslash \Omega_{2 \varepsilon}\right)\right) .
$$

Since $\mathcal{H}^{N-1}\left(\partial E \cap\left(\Omega \backslash \Omega_{2 \varepsilon}\right)\right) \rightarrow 0$ as $\varepsilon \rightarrow 0$, using a diagonal argument, we deduce the thesis of the corollary.

Corollary 4.10. Let $\Omega \subset \mathbb{R}^{N}$ have a continuous boundary, and let $E$ be a polyhedral set in $\Omega$ in the previous sense. Then,

$$
\left(\Gamma-\lim _{h \rightarrow 0} J_{h}(\cdot, \Omega)\right)\left(\chi_{E}\right)=J\left(\chi_{E}, \Omega\right) .
$$




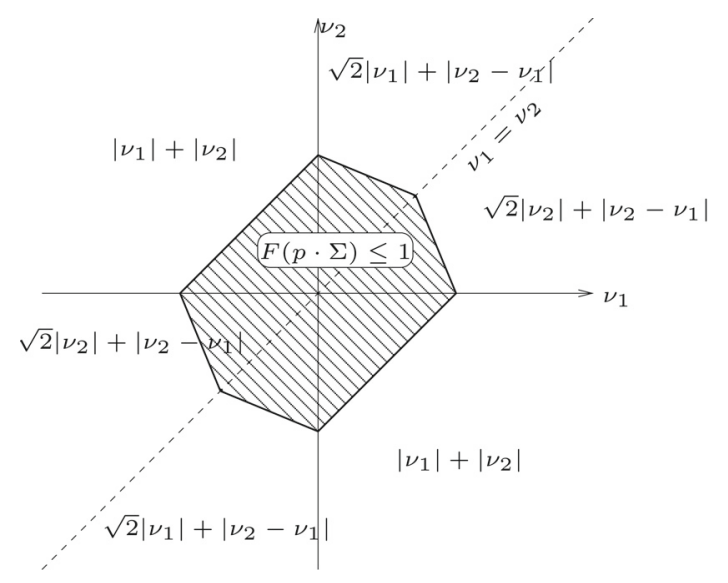

FIGURE 1. Values of $F$ given by (5.1).

Proof. It follows from (4.12) (which has been shown in Sect. 4.1) and from Proposition 4.9.

Now, we are in a position to show that (4.13) holds.

Proposition 4.11. Let $\Omega$ be a bounded open set of $\mathbb{R}^{N}$ with Lipschitz boundary, and let $E \subset \Omega$ be a set with finite perimeter in $\Omega$. Then for every $h>0$ there exists $E_{h}$ with $\chi_{E_{h}} \in V_{h}(\Omega)$, such that $\chi_{E_{h}} \rightarrow \chi_{E}$ in $L^{1}(\Omega)$ as $h \rightarrow 0$ and

$$
\limsup _{h \rightarrow 0} J_{h}\left(\chi_{E_{h}}, \Omega\right) \leq J\left(\chi_{E}, \Omega\right) .
$$

Proof. Since $\Omega$ is Lipschitz, we can extend $E$ outside of $\Omega$ into a finite-perimeter subset of $\mathbb{R}^{N}$ (still denoted $E$ ) such that $\left|D \chi_{E}\right|(\partial \Omega)=0$. Then, standard approximation arguments show that there exists a sequence of polyhedral sets $G^{n}$ such that $\chi_{G^{n}}$ converges to $\chi_{E}$ strongly in $L^{1}\left(\mathbb{R}^{N}\right)$, and with

$$
\lim _{n \rightarrow \infty}\left|D \chi_{G^{n}}\left(\mathbb{R}^{N}\right)\right|=\left|D \chi_{E}\right|\left(\mathbb{R}^{N}\right) .
$$

This can be seen, for instance, by approximating $\chi_{E}$ by smooth functions (by convolution) and then approximating these smooth functions by piecewise linear functions, such as "P1" finite-elements. Then, an appropriate thresholding of these functions provides the sequence $G^{n}$. The Reshetnyak continuity theorem (see Sect. 2), and $\left|D \chi_{E}\right|(\partial \Omega)=0$ yield

$$
\lim _{n \rightarrow \infty} J\left(\chi_{G^{n}}, \Omega\right)=J\left(\chi_{E}, \Omega\right) .
$$

By Proposition 4.9, we know that for each $n$ there exists $G_{h}^{n}$ converging to $G^{n}$, such that $\lim _{\sup } \operatorname{su}_{h \rightarrow 0} J_{h}\left(G_{h}^{n}, \Omega\right) \leq$ $J\left(\chi_{G^{n}}, \Omega\right)$. We construct the family $\left(E_{h}\right)_{h>0}$ from the $G_{h}^{n}$, by a diagonal argument as follows. For every $n$ there exists $h_{n}$ such that $h_{n} \downarrow 0$ as $n \rightarrow \infty$ such that for every $h \leq h_{n}$ we have $\left\|\chi_{G_{n}^{n}}-\chi_{G^{n}}\right\|_{L^{1}(\Omega)} \leq 1 / n$ and $J_{h}\left(\chi_{G_{h}^{n}}, \Omega\right) \leq J\left(\chi_{G^{n}}, \Omega\right)+1 / n$. If we set $E_{h}:=G_{h}^{n}$ for $h_{n+1}<h \leq h_{n}$, the result follows.

\section{EXAMPLES}

Let us describe a few cases. First of all, the standard nearest-neighbor interaction on a square grid corresponds to the situation where $\Sigma=\{(0,0),(1,0),(0,1)\}$ and, for $u \in \mathbb{R}^{\Sigma}, F(u)=|u(1,0)-u(0,0)|+|u(0,1)-u(0,0)|$. It is obvious, in this case (as in any other case where $F$ is a sum of pair interactions) that the $\Gamma$-limit of $J_{h}$ is the anisotropic total variation given by (1.6), in this case, $\int_{\Omega}|D u|_{1}$ where $|p|_{1}=\left|p_{1}\right|+\left|p_{2}\right|$ is the 1-norm in $\mathbb{R}^{2}$. 
Less trivial situations are when $F$ cannot be reduced to a sum of pair interactions, such as, still with the same set $\Sigma$, the functions $F$ defined by $F\left(0_{\Sigma}\right)=F\left(1_{\Sigma}\right)=0, F\left(1_{\Sigma}-u\right)=F(u)$ for any $u \in\{0,1\}^{\Sigma}$, and

$$
F\left(\begin{array}{ll}
0 & \\
0 & 1
\end{array}\right)=1, \quad F\left(\begin{array}{ll}
1 & \\
0 & 0
\end{array}\right)=1, \quad F\left(\begin{array}{ll}
1 & \\
0 & 1
\end{array}\right)=\sqrt{2} .
$$

This $F$ can also be checked to be submodular. Now, the limit density is given by

$$
\nu=\left(\nu_{1}, \nu_{2}\right) \mapsto F\left(\begin{array}{ll}
\nu_{2} & \\
0 & \nu_{1}
\end{array}\right),
$$

see Figure 1 for the expression of $F$, and where we also have plotted the shape of the "Wulff shape" $F(p \cdot \Sigma) \leq 1$.

Notice that in this case, we have chosen $F(\theta)=\sqrt{\left(\theta_{1,0}-\theta_{0,0}\right)^{2}+\left(\theta_{0,1}-\theta_{0,0}\right)^{2}}$ for $\theta \in \Sigma^{\{0,1\}}$ : however, clearly, we get a limit energy which is not $\int_{\Omega}|D u|$ (which would be the $\Gamma$-limit of $u \mapsto h \sum_{i, j}$ $\left.\sqrt{\left(u_{i+1, j}-u_{i, j}\right)^{2}+\left(u_{i, j+1}-u_{i, j}\right)^{2}}\right)$ but an anisotropic (crystalline) total variation. This is of course due to the fact that the latter does not satisfy the generalized coarea formula.

Acknowledgements. A. C. is supported by ANR, program "MICA", grant ANR-08-BLAN-0082 and by the CNRS. A. G. is supported by the Italian Ministry of University and Research, project "Variational problems with multiple scales" 2006. L. L. was partially supported by the Istituto Nazionale di Alta Matematica (Roma, Italy) and by the Università Cattolica del Sacro Cuore (Brescia, Italy) during the permanence of the author at the Centre de Mathématiques Appliquées of the École polytechnique (Palaiseau, France).

\section{REFERENCES}

[1] R.K. Ahuja, T.L. Magnanti and J.B. Orlin, Network flows, Theory, algorithms, and applications. Prentice Hall Inc., Englewood Cliffs, USA (1993).

[2] L. Ambrosio, N. Fusco and D. Pallara, Functions of bounded variation and free discontinuity problems, Oxford Mathematical Monographs. The Clarendon Press Oxford University Press, New York, USA (2000).

[3] Y. Boykov and V. Kolmogorov, Computing geodesics and minimal surfaces via graph cuts, in International Conference on Computer Vision (2003) 26-33.

[4] Y. Boykov and V. Kolmogorov, An experimental comparison of min-cut/max-flow algorithms for energy minimization in vision. IEEE Trans. Pattern Anal. Mach. Intell. 26 (2004) 1124-1137.

[5] A. Braides, $\Gamma$-convergence for beginners, Oxford Lecture Series in Mathematics and its Applications 22. Oxford University Press, Oxford, UK (2002).

[6] A. Chambolle and J. Darbon, On total variation minimization and surface evolution using parametric maximum flows. Int. J. Comput. Vis. 84 (2009) 288-307.

[7] W.H. Cunningham, On submodular function minimization. Combinatoria 5 (1985) 185-192.

[8] G. Dal Maso, An introduction to $\Gamma$-convergence, Progress in Nonlinear Differential Equations and their Applications 8. Birkhäuser Boston Inc., Boston, USA (1993).

[9] H. Federer, Geometric measure theory. Springer-Verlag New York Inc., New York, USA (1969).

[10] E. Giusti, Minimal surfaces and functions of bounded variation, Monographs in Mathematics 80. Birkhäuser Verlag, Basel, Switzerland (1984).

[11] D.M. Greig, B.T. Porteous and A.H. Seheult, Exact maximum a posteriori estimation for binary images. J. R. Statist. Soc. B 51 (1989) 271-279.

[12] S. Iwata, L. Fleischer and S. Fujishige, A combinatorial, strongly polynomial-time algorithm for minimizing submodular functions, in Proceedings of the 32nd annual ACM symposium on Theory of computing, ACM (2000) 97-106.

[13] L. Lovász, Submodular functions and convexity, in Mathematical programming: the state of the art (Bonn, 1982), Springer, Berlin, Germany (1983) 235-257.

[14] J.C. Picard and H.D. Ratliff, Minimum cuts and related problems. Networks 5 (1975) 357-370.

[15] A. Schrijver, A combinatorial algorithm minimizing submodular functions in strongly polynomial time. J. Comb. Theory (B) 80 (2000) 436-355.

[16] A. Visintin, Nonconvex functionals related to multiphase systems. SIAM J. Math. Anal. 21 (1990) 1281-1304.

[17] A. Visintin, Generalized coarea formula and fractal sets. Japan J. Indust. Appl. Math. 8 (1991) 175-201. 